\title{
DIE RÖMER UND IHRE HOSPITÄLER
}

\author{
Beobachtungen zu den Trägergruppen \\ der Spitalsgründungen in Rom (13.-15. Jahrhundert)
}

Die wissenschaftliche Literatur zur Geschichte des Hospitals ist kaum noch zu überblicken ${ }^{1}$. Unter der Vielzahl methodischer Ansätze für die Erforschung mittelalterlicher Hospitäler ist die Prosopographie eine Disziplin, die noch lange nicht ausgeschöpfte Erkenntnismöglichkeiten birgt. Ihr Potential soll an dieser Stelle für die Frage nach den Gründungsmotiven in der römischen Hospitallandschaft genutzt werden. Insbesondere sollen die Interessen und die sozialen und politischen Hintergründe beleuchtet werden ${ }^{2}$, die mit den Hospitalsgründungen in Rom vom 13. bis 15. Jahrhundert verknüpft waren. Dabei haben die römischen Spitäler und die sie oft tragenden Konfraternitäten schon seit einiger Zeit das Interesse der Forschung auf sich gezogen, so daß man eigentlich kein Neuland betritt ${ }^{3}$. Trotzdem sind gerade die genauen Entste-

${ }^{1}$ Zur breiten Literatur zum Hospitalswesen verweise ich auf die Forschungsberichte von Gisela Drossbach, François-Olivier Touati und Thomas Frank in diesem Tagungsband.

${ }^{2} \mathrm{Vgl}$. zu diesem spezifischen Interesse demnächst den Band Sozialgeschichte mittelalterlicher Hospitäler, hg. v. Neidhart BULST, Karl-Heinz SPIESS, Sigmaringen (Vorträge und Forschungen des Konstanzer Arbeitskreises) (im Druck). Anregend ist auch - obgleich nicht direkt mit Spitalbruderschaften befaßt - Ludwig REMLING, Sozialgeschichtliche Aspekte des spätmittelalterlichen Bruderschaftswesens in Franken, in: Peter JOHANEK (Hg.), Einungen und Bruderschaften in der spätmittelalterlichen Stadt, Köln u.a. 1993 (Städteforschung/A, 32), S. 149-169, bes. S. 160ff.; Ralf LUSIARDI, Stifung und städtische Gesellschaft. Religiöse und soziale Aspekte des Stiftungsverhaltens im spätmittelalterlichen Stralsund, Berlin 2000 (Stiftungsgeschichten, 2) beschäftigt sich am Beispiel Stralsunds in sozialhistorischer Perspektive allgemein mit spätmittelalterlichen Stiftungen zwischen caritas und Sozialfürsorge. Seine Studie ist der zweite Band der von Michael Borgolte herausgegebenen Reihe zum mittelalterlichen Stiftungswesen.

${ }^{3}$ Für die großen Linien der Entwicklung der römischen Hospitallandschaft im 14. und 15. Jahrhundert vgl. Anna ESPOSITO, Gli ospedali romani tra iniziative laicali e politica pontificia (secc. XIII-XV), in: J. GRIECO, Lucia SANDRI (Hg.), Ospedali e città. L'Italia del Centro-Nord, XIII-XVI secolo. Atti del convegno internazionale di studio tenuto dall'Istituto degli Innocenti e Villa i Tatti (Firenze 27-28 aprile 1995), Florenz 1997, S. 233-251 (mit weiterer Bibliographie); DIES., Amministrare la devozione. Note dai libri sociali delle confraternite romane (secc. XV-XVI), in: II buon fedele. Le confraternite tra medioevo e prima età moderna $=$ Quaderni di storia religiosa 5 (1998) S. 195-223. 
hungsumstände der Hospitäler in Rom oft noch nicht geklärt. Der Wortlaut der Hospitalstatuten (in Rom nur greifbar in der Regula hospitalis Sancti Spiritus, die eigentlich eine Ordensregel ist) ${ }^{4}$ und der Statuten der ein Hospital tragenden Bruderschaften ${ }^{5}$ ist meist zu allgemein gehalten, als daß die - hier im Vordergrund stehende - soziale Einbindung in das städtische Umfeld auf Anhieb deutlich würde. Daneben eröffnet die Hospitallandschaft in Rom auch interessante Perspektiven für die Typologisierung des Begriffs "Hospital«. Denn es macht einen Unterschied, ob ein Hospital von einem Orden (einem alten oder neuen), von einem Laien oder einem Geistlichen, oder von einer Bruderschaft (wobei man auch diese differenzieren kann) gegründet und unterhalten wurde ${ }^{6}$. Eng mit den Entstehungsumständen sind auch die spezifische Ausrichtung und der Zweck, dem ein Hospital dienen sollte, verknüpft. Hier war oft entscheidend, ob man - abgesehen von dem spirituellen Wert, den die Errichtung einer karitativen Institution an sich besaß - selbst materieller Nutznießer seiner eigenen Gründung werden wollte oder ob sich das neue Haus an einen breiteren Nutzerkreis wandte. Für all diese Aspekte ist Rom ein

${ }^{4}$ Zur Typologie und Definition von Hospitalstatuten siehe Michel MOLLAT, Complexité et ambiguïté des institutions hospitalières: les statuts d'hôpitaux (les modèles, leur diffusion et leur filiation), in: Giorgio POLITI, Mario ROSA, Franco DELLA PERUTA (Hg.), Timore e carita. I poveri nell'Italia moderna, Atti del convegno »Pauperismo e assistenza negli antichi stati italiani« (Cremona, 28-30 marzo 1980), Cremona 1982 (Annali della Biblioteca Statale e Libreria Civica di Cremona, 27-30), S. 3-12 und jetzt den Beitrag von Gisela Drossbach im vorliegenden Band.

${ }^{5}$ Vgl. Paola PAVAN, Gli statuti della Società dei Raccomandati del Salvatore ad Sancta Sanctorum (1331-1496), in: Archivio della Società Romana di Storia Patria 101 (1978) S. 35-96 und Silvana Di MATTIA SPIRITO, Assistenza e carità ai poveri in alcuni statuti di confraternite nei secoli XV-XVI, in: Ricerche per la storia religiosa in Roma 5 (1984) S. 137-154. Die Hintergründe der Rekrutierung der Mitglieder sind in der Regel nicht ersichtlich in den Statuten, die von ihnen auch kein soziales Profil entwerfen, sondern vorrangig ihre moralische Eignung in den Vordergrund stellen (wiederkehrend ist der Ausschluß von Wucherern und solchen, die im Konkubinat lebten): PAVAN, Gli statuti, S. 47. Natürlich garantierte auch die Kooption des Sohnes eines verstorbenen Mitglieds (ibid. S. 47f.) die soziale Geschlossenheit, ohne aber über deren Charakteristika Aufschluß zu geben. Instruktiver sind die Statuten dagegen für das Verhältnis der Laien zu den Klerikern unter den Mitbrüdern: ibid. S. 49f. Eine Auswertung von Statuten mit vielen Facetten - hier die der Bruderschaft des Campo Santo Teutonico - bietet Knut SCHULZ, Confraternitas Campi Sancti de Urbe. Die ältesten Mitgliederverzeichnisse (1500/01-1536) und Statuten der Bruderschaft, Rom u.a. 2002 (Römische Quartalschrift. Supplementbd., 54), S. 38-48 (Edition: S. 154-164).

${ }^{6}$ Nach Siegfried REICKE, Das deutsche Spital und sein Recht im Mittelalter. Erster Teil: Das deutsche Spital. Geschichte und Gestalt, Stuttgart 1932 (Kirchenrechtliche Abhandlungen, 111) (unveränd. Nachdruck Amsterdam 1961), hier I, S. 48-92 ist zu unterscheiden zwischen einer geistlich ausgerichteten Spitalbruderschaft (aus der - wie im Falle des S. Spirito in Sassia - ein Orden hervorgehen konnte) und einer weltlichen Spitalbruderschaft, die der materiellen Unterstützung eines Spitals diente, ohne daß die beteiligten Laien ihren Beruf aufgaben und ein gemeinsames Leben führten. 
hervorragendes Untersuchungsfeld, zumal die Stadt nach dem um $1325 \mathrm{zu}$ datierenden Katalog von Turin 25 z.T. sehr unterschiedliche Hospitäler umfaßte (und diese Zahl ist noch leicht aufzustocken) ${ }^{7}$. Was diese Vielfalt an Krankenhospizen betrifft, war Rom unter den italienischen Kommunen kein Einzelfall $^{8}$, die römische Sozialfürsorge weist aber einige Besonderheiten auf.

In Ermangelung direkter Quellen für unsere Fragestellung, kommt dem Historiker die prosopographische Methode zustatten. Denn sie führt dann weiter, wenn man wenig über das einzelne Individuum (sei es nun in unserem Fall der oder die Gründer oder der oder die Nutzer und Träger eines Hospitals) weiß und auf eine kollektive Gesamtschau angewiesen ist. Und für diesen methodischen Ansatz haben sich in Rom in den Nekrologien, Anniversarien- ${ }^{9}$ und

${ }^{7}$ Zur Zahl im Katalog von Turin: Christian HuelseN, Le chiese di Roma nel Medio Evo. Cataloghi ed appunti, Florenz 1927 (unveränd. Nachdruck Rom 2000), S. 42f. Für einen ersten Gesamtüberblick über die Hospitallandschaft in Rom vgl. Alessandro CANEZZA, Gli arcispedali di Roma nella vita cittadina nella storia e nell'arte, Rom 1933; Mariano DA ALATRI, Il medio evo, in: Vincenzo MONACHINO ( $\mathrm{Hg}$.), La carità cristiana in Roma, Bologna 1968 (Roma cristiana, 10), S. 123-187, hier S. 139-179; Anna EsPOSITO, Accueil et assistance à Rome, in: Médiévales 40 (2001) S. 29-41 (mit weiterer Bibliographie). Unbefriedigend, da nur alte Literatur aufarbeitend und lückenhaft, ist das Repertorium zu 58 im 15. Jahrhundert nachgewiesenen römischen Hospitälern, die unsystematisch den Kategorien ON (Ospedale Nazionale), OP (Ospedale Pubblico [darunter fallen die Spitäler der Bruderschaften]), OA (Ospedale delle Arti [mit nur drei Nummern!]) zugeordnet werden, in Flavia COLONNA, Distribuzione urbana e tipologie degli edifici assistenziali, in: Giorgio SIMONCINI (Hg.), Roma. Le trasformazioni urbane nel Quattrocento, II: Funzioni urbane e tipologie edilizie, Florenz 2004 (L'Ambiente storico. Studi di storia urbana e del territorio, 11), S. 159-188. Für die Anfänge des mittelalterlichen Hospitalwesens (Diakonien, Xenodochien), die eng mit der Aufnahme und Versorgung von Pilgern verknüpft waren, vgl. Debra J. BIRCH, Pilgrimage to Rome in the Middle Ages: Continuity and Change, Woodbridge 1998, S. 123-149. Zahlenmäßig nicht zu erfassen sind die zahlreichen privaten Hospitalsgründungen, von denen man oft nur aus Testamenten weiß und die meist nur kurz bestanden, sofern sie nicht von einem größeren Hospital einverleibt wurden: vgl. Anna ESPOSITO. l.e confraternite romane tra arte e devozione: persistenze e mutamenti nel corso del $\mathrm{XV}$ secolo. in: Amold ESCH, Christoph L. FROMMEL (Hg.), Arte, committenza ed economia a Roma e nelle corti del Rinascimento (1420-1530). Atti del convegno internazionale, Roma 24-27 ottobre 1990 Turin 1995 (Piccola Biblioteca Einaudi, 630), S. 107-120, hier S. 109; Isa LORI SANFILIPPO, La Roma dei romani. Arti, mestieri e professioni nella Roma del Trecento. Rom 2001 (Nuovi studi storici, 23), S. 419.

${ }^{8}$ Florenz besaß um 1450 dreiunddreiBig "spedali« (nur zwei davon waren speziell für Pilger ausgerichtet): John HENDERSON, The Hospitals of Late Medieval Florence. A Preliminary Survey, in: Lindsay GRANSHAW and Roy PORTER (Hg.), The Hospital in History, London 1989, S. 63-92; DERS., Piety and Charity in Late Medieval Florence, Oxford u.a. 1994, S. 375 .

${ }^{9}$ Zur Definition und Abgrenzung zwischen Nekrologen und Anniversarien, zu ihrem Quellenwert und zu den Problemen mit ihrem Umgang vgl. Nicolas HuYgheBaERT, Les documents nécrologiques, Turnhout 1972 (Typologie des sources du moyen âge occidental, 4), bes. S. 33ff.; Jean-Loup LEMAITRE, Les documents nécrologiques. Mise à jour du fascicule $n^{\circ}$ 4, Turnhout 1985; Peter-Johannes SCHULER, Das Anniversar. Zu Mentalität und Famili- 
Bruderschaftsbüchern für vier interessante Hospitalstypen hervorragende Quellen erhalten. Hier geht es allerdings nicht darum, Zahlen und Prozente zu präsentieren ${ }^{10}$. Für quantitative Analysen fehlen die Vorarbeiten, ist ja schon die vor gut 100 Jahren von Pietro Egidi vorgelegte Edition dieser Texte in vielerlei Hinsicht unbefriedigend ${ }^{11}$ und ihre - möglichst computergestützte - Erschließung über Indizes überfällig ${ }^{12}$. Die vier Hospitäler, die jetzt vorgestellt werden, decken zwar nicht das ganze Spektrum der römischen Spitalsgründungen ab, vermitteln aber doch einen ersten Überblick über die wichtigsten karitativen Stiftungstypen, die allerdings nicht primär nach ihren Funktionen, sondern nach den Trägergruppen untersucht und definiert werden sollen ${ }^{13}$. Weitgehend unbeachtet müssen indes einige Aspekte bleiben, die ebenfalls mit denselben Quellen - sowie mit den Statuten und etwa vorhandenen Testamen$\operatorname{ten}^{14}-\mathrm{zu}$ untersuchen wären, wie zum Beispiel religiöse Vorstellungen, Mentalität, Verwaltung und Personal des Hospitals oder wirtschaftliche Aspekte ${ }^{15}$.

enbewußtsein im Spätmittelalter, in: DERS. (Hg.), Die Familie als sozialer und historischer Verband. Untersuchungen zum Spätmittelalter und zur frühen Neuzeit, Sigmaringen 1987, S. $67-117$, bes. S. $70 \mathrm{ff}$.

${ }^{10} \mathrm{Vgl}$. zu den Möglichkeiten der Aufarbeitung als Beispiel Beate S. GROS, Das Hohe Hospital (ca. 1178 bis 1600). Eine prosopographische und sozialgeschichtliche Untersuchung, Münster 1999 (Urkunden-Regesten der Soester Wohlfahrtsanstalten, 5).

11 Vorweg sei auf das Manko hingewiesen, daß in der Regel - allerdings angesichts der Menge verständlich - nur Materialien bis zu dem recht willkürlich gewählten Stichjahr 1500 aufgenommen wurden. Pietro EGIDI, Necrologi e libri affini della Provincia Romana. Necrologi della città di Roma, Rom 1908-1914 (Fonti per la storia d'Italia, 44/45). Zum Gesamtkonzept, das eigentlich auch einen dritten Band (zu Latium) umfassen sollte, siehe DERS.. I necrologi e i libri affini della provincia romana nel medio evo, in: Bullettino dell'Istituto Storico Italiano per il medio evo e Archivio Muratoriano 25 (1904) S. 85-92 und die Rezension zum ersten Band der Edition von Vincenzo FEDERICl, in: Archivio storico italıano. 5 ser. 44 (1909) S. 419-424.

${ }^{12}$ Eine solche quantitative Analyse der Mitglieder liegt nur im Falle der Bruderschaft des Campo Santo Teutonico vor, deren Mitgliederlisten allerdings erst 1501 einsetzen: SCHULZ, Confraternitas Campi Sancti (wie Anm. 5), bes. S. 58-65 (analysiert werden die Einträge von 1501-1536). Diese verdienstvolle Arbeit zeigt auch anschaulich die methodischen Schwierigkeiten (z.B. das Problem der Doppelnennungen, Besonderheiten der Registrierung etc.), die eine solche vertiefte Analyse erfordert. Ibid. S. 121-140 werden die technischen Voraussetzungen der computergestützten Edition der Mitgliederverzeichnisse (S. 165-305) und Registererstellung erläutert. Für weitere technische Anregungen sei auf die Zeitschrift Fundus - Forum für Geschichte und ihre Quellen (http://webdoc.gwdg.de/edoc/p/fundus/ index.html) verwiesen.

${ }^{13}$ Zur breiten Typologie der mittelalterlichen Hospitäler vgl. an dieser Stelle nur den Beitrag von Gisela Drossbach in diesem Band sowie Michael MATHEuS (Hg.), Funktions- und Strukturwandel spätmittelalterlicher Hospitäler im europäischen Vergleich, Stuttgart 2005 (Geschichtliche Landeskunde, 56).

${ }^{14}$ Für Rom müßte außerdem noch die Beziehung zwischen den mitunter vorhandenen Testamenten und den Einträgen in die Anniversarlisten untersucht werden: vgl. dazu sehr anregend Wolfgang E. WAGNER, Von der Stiftungsurkunde zum Anniversarbucheintrag. 


\section{Zentrum eines Hospitalordens und das `päpstliche‘ Hospital schlechthin: das Hospital von Santo Spirito in Sassia}

Es wird nicht überraschen, wenn der Überblick mit einem Hospital beginnt, das - wie nicht zuletzt Gisela Drossbach herausgestrichen hat ${ }^{16}$ - als das 'päpstlicheく Hospital schlechthin gelten kann ${ }^{17}$. Die Päpste nannten es hospitale nostrum und überhäuften es mit Privilegien. Dieses enge Verhältnis zwischen Kurie und Hospital war von Innozenz III. grundgelegt worden, der nicht zuletzt den Vorzug der räumlichen Nähe des Hospitals zur Peterskirche erkannt hatte, die es zu dem bevorzugten Krankenhaus der Pilger und Kurienangehörigen prädestinierte. Diese beiden Gruppen - Auswärtige und Kuriale - sollten auch in Zukunft das soziale Umfeld dieses Hauses bestimmen (wo sie sich auch einquartieren konnten), während die Römer selbst - und die römische Kommune - eine zunächst untergeordnete, wenn auch nicht zu unterschätzende Rolle spielten ${ }^{18}$. Der Grund hierfür ist in den Umständen der Gründung zu suchen, die nicht auf eine stadtrömische, sondern - wie gesagt - auf eine päpstliche Initiative zurückgeht und die mit einem Orden verbunden ist, und zwar mit dem Heilig-Geist-Orden, dessen Ursprünge in Südfrankreich zu suchen sind. Das von vorneherein in großen Dimensionen geplante Hospital, das Hunderte von Kranken versorgen sollte, stellte nicht nur für Rom ein Novum

Beobachtungen zur Anlage des Liber oblationum et anniversarionum (1442-ca. 1480) im Wiener Schottenkloster, in: Michael BORGOLTE, Stiftungen und Stiftungswirklichkeiten. Vom Mittelalter bis zur Gegenwart (Stiftungsgeschichten, 1), Berlin 2000, S. 145-170.

${ }^{15}$ Hierzu sei auf den Beitrag von John Henderson im vorliegenden Band und die weiterführende Literatur in der Anm. 9 sowie - für die religiösen Beweggründe - auf die Sammelbände Esperienze religiose e opere assistenziali nei secoli XII e XIII, hg, von Grado G. MERLO, Turin 1988 und La conversione alla povertà nell'Italia dei secoli XII-XIV. Atti del XXVII convegno storico internazionale, Todi, 14-17 ottobre 1990, Spoleto 1991 (Accademia Tudertina. Centro di studi sulla spiritualità medievale. Nuova serie, 4) verwiesen.

${ }^{16} \mathrm{Vgl}$. hier nur Gisela DrossBaCH, >Regularis ordo ... per nos institutus esse dinoscitur. Zum Gründungsvorgang des Spitalordens vom Heiligen Geist durch Innocenz III., in: Kenneth Pennington u.a. (Hg.), Proceedings of the Tenth International Congress of Medieval Canon Law, Syracuse, New York, 13-18 August 1996, Città del Vaticano 2001, S. 387-404.

${ }^{17}$ Zur Geschichte des Hospitals vgl. Pietro DE ANGELIS, L'ospedale di S. Spirito in Saxia, 2 Bde., Rom 1960-1962; L'Antico Ospedale di Santo Spirito. Dall'istituzione papale alla sanità del terzo millenio, I-II, in: Il Veltro, 45/5-6 (2001), 46/1-4 (2002); Andreas REHBERG, I papi, l'ospedale e l'ordine di S. Spirito nell'età avignonese, in: Archivio della Società Romana di Storia Patria 124 (2001) S. 35-140; Gisela DrossBaCH, Christliche caritas als Rechtsinstitut. Hospital und Orden von Santo Spirito in Sassia (1198-1378) (Kirchenund Staatskirchenrecht, 2), Paderborn 2005.

${ }^{18}$ Die Kommune gewährte - wenn auch angesichts des großen Landbesitzes des Hospitals mitunter widerstrebend - Steuer- und Abgabenfreiheit: Sigismondo MALATESTA, Statuti delle gabelle di Roma, Rom 1885, S. 122-134 (1354 Sept. 16, 1368 Okt. 3, 1385 März 16, 1390 Jan. 10). Vgl. auch die Angabe zu den Stadtstatuten in Anm. 157. 
dar und nahm das anderenorts erst allmählich einsetzende Bestreben nach Zentralisierung und Rationalisierung vorweg ${ }^{19}$. Es dauerte nicht lange, daß das reiche Hospital auch die Aufmerksamkeit der Römer gewann, kamen sie doch allein schon über wirtschaftliche Interessen mit ihm in Kontakt. Als Großgrundbesitzer in Latium (und darüber hinaus) und als Eigentümer zahlreicher Häuser in Rom zog es bald viele Pächter aus der Tiber-Stadt an. Das Hospital war außerdem direkter Nachbar eines der großen Baronalgeschlechter Roms, und zwar der Orsini, denen es im späten 13. und im 14. Jahrhundert gelang, allein vier Kardinalprotektoren des Ordens zu stellen. Der Rückhalt in einem dominanten Adelsgeschlecht war eine zweischneidige Sache, geriet S. Spirito so doch zwangsläufig in den Teufelskreis der die Stadt Rom belastenden Fraktionskämpfe. Das Hospital war auch Pressionen ausgesetzt, dem großen Orsini-Clan einige Ländereien zuzuschanzen ${ }^{20}$.

Die Ordensregel des S. Spirito ${ }^{21}$ beschreibt natürlich den Krankendienst in vielen Einzelheiten, macht aber wenige Aussagen zur Integration des Hospitals in das römische Umfeld. Mehr Hinweise ergeben sich aus dem liturgischen Gebetsgedenken (memoria) ${ }^{22}$, das in Santo Spirito in Sassia besonders gepflegt wurde. Schon die Ordensregel erwähnt das Nekrologium (kalendari$u m$ ), das man mit dem noch heute erhaltenen sog. Liber annualium identifizieren kann, ein nach Monat und Tag geordnetes Verzeichnis der Mitglieder und Wohltäter, das in der ersten Hälfte des 13. Jahrhunderts einsetzt und auch einige Stiftungen mitnotiert ${ }^{23}$. Unter den hier erfaßten Personen dominieren die

${ }^{19} \mathrm{Vgl}$. zu den genannten Phänomenen, die keineswegs von vorneherein von der Kirche abgelehnt wurden, zum Beispiel - für die Toskana und Venedig - Marvin B. BECKER, Aspects of Lay Piety in Early Renaissance Florence, in: Charles TRINKAUS mit Heiko A. OBERMAN (Hg.), The Pursuit of Holiness in Late Medieval and Renaissance Religion. Papers from the University of Michigan Conference, Leiden 1974 (Studies in Medieval and Reformation Thought, 10), S. 177-199, hier S. 187; Brian PULLAN, Rich and Poor in Renaissance Venice. The Social Institutions of a Catholic State, to 1620, Oxford 1971 (italienische Übersetzung: La politica sociale della Repubblica di Venezia, 1500-1620, Rom 1982) S. 197ff. und - für die Lombardei - Giuliana ALBINI, Città e ospedali nella Lombardia medievale, Bologna 1993 (Biblioteca di storia urbana, 8), S. 122, 114ff, 140ff.

${ }^{20}$ Vgl. zur Rolle der Orsini ReHBERG, I papi, l'ospedale (wie Anm. 17), bes. S. 77f., 89-93.

${ }^{21}$ Vgl. Francesco LA CAVA, Liber regulae S. Spiritus (Studi di Storia della Medicina, o. Bd.), Mailand 1947 und DROSSBACH, Christliche caritas (wie Anm. 17), S. 354-431.

${ }^{22}$ Vgl. hierzu den Beitrag von Thomas Frank in diesem Band.

${ }^{23}$ Rom, Archivio di Stato (hinfort ASR), Ospedale di S. Spirito, 72 (vormals: Rom, Biblioteca Lancisiana, 262; vgl, eine im 15. Jahrhundert angelegte Kopie: ASR, Ospedale di S. Spirito, 9), ed. EGIDI, Necrologi (wie Anm. 11), I, S. 109-111 (zur Anlage und Datierung der Schrift Ende des 13. Jahrhunderts), 112-165 (Edition); Marc DYKMANS, Les obituaires romains. Une définition suivie d'une vue d'ensemble, in: Studi medievali, ser. 3a, 19/2 (1978) S. 591-652, hier S. $627 \mathrm{f}$. 
Ordensmitglieder, die man an der Bezeichnung frater oder soror erkennt. ${ }^{24}$ Vor allem Italiener - darunter aber nur wenige Römer - bilden das Personal des Krankenhauses ${ }^{25}$. Gelegentlich trifft man auf Ordensmitglieder von jenseits der Alpen ${ }^{26}$. Die Namen der Päpste (Innozenz III., Innozenz IV., Alexander IV., Hadrian V. und Nikolaus III. $)^{27}$ und einiger Kardinäle und Bischöfe ${ }^{28}$ sowie von Kurialen und Geistlichen ${ }^{29}$ unterstreichen den engen Bezug zum Papsttum und zur Kurie. Selbst königliche Gönner - allen voran König Johann ohne Land von England ${ }^{30}$ - fehlen nicht. Mehr Einheimische trifft man erst unter den Namen an, die im 14. Jahrhundert eingetragen wurden, als infolge des Umzugs nach Avignon die Dominanz der Kurie im Alltag des Hospitals nachlie $\beta^{31}$. Diese Römer waren oft oblati und Wohltäter des Hospitals, über die auch andere Quellen (z.B. Testamente) unterrichten. Einige Namen gehören zur politischen Klientel der Orsini, wobei aber nicht alle Wohltäter des Hospitals auch im Nekrolog verzeichnet sind, denkt man nur an die dort fehlenden Kardinäle Giacomo Stefaneschi und Giovanni Boccamazza. ${ }^{32}$ Andere vornehme Geschlechter mit Orsini-Kontakten waren die Conti, Normanni, Malabranca, Frangipane sowie die Tartari $^{33}$. Insgesamt fallt auf, daß im

${ }^{24}$ Für den weiblichen Zweig des Heiliggeist-Ordens vgl. B. RANO, Ospitaliere di Santo Spirito, in: Dizionario degli Istituti di Perfezione, VI, Rom 1980, Sp. 988-993.

${ }^{25} \mathrm{Zu}$ einem solchen mutmaßlichen Römer, frater Jacobus Porcarii, und zu Contius domini Mathei aus Trastevere vgl. EGIDI, Necrologi (wie Anm. 11), I, S. 116.

${ }^{26} \mathrm{Vgl}$. Andreas REHBERG, Die fratres von jenseits der Alpen im römischen Hospital S. Spirito in Sassia, in: Uwe ISRAEL (Hg.), Vita communis und ethnische Vielfalt. Multinational zusammengesetzte Klöster im Mittelalter, Berlin 2006 (Vita regularis, 29), S. 97-155.

${ }^{27}$ Siehe EgIDI, Necrologi (wie Anm. 11), I, S. 132, 142, 148, 160, 162.

${ }^{28} \mathrm{Vgl}$. nur ibid. S. 140, 142, 152, 154, 158.

${ }^{29} \mathrm{Zu}$ Mitgliedern der päpstlichen capella vgl. ibid. S. 126, 148. Vgl. zu Kanonikern von St. Peter, die allein schon in Anbetracht der geographischen Nähe ein enges Verhältnis zum Hospital unterhielen: ibid. S. 118, 130. Andere Beispiele ibid. S. 126.

${ }^{30}$ Ibid. S. 154. Die Könige von Neapel aus dem Hause Anjou leiten in das 14. Jahrhundert über: ibid. S. 154.

${ }^{31}$ Die Edition Egidis unterscheidet die Einträge nach dem 13., 14. und 15. Jahrhundert. Die Abgrenzung der ersten beiden Jahrhunderte ist aber aufgrund der fehlenden Jahresangaben nicht immer exakt. Zu dem ibid. I, S. 126 in der Rubrik »Sec. XIII« verzeichneten Francesco Hominisdei, der in Wirklichkeit 1324 gestorben ist (wenn es sich nicht um einen Namensvetter handelt), vgl. Andreas REHBERG, Die Kanoniker von S. Giovanni in Laterano und S. Maria Maggiore im 14. Jahrhundert. Eine Prosopographie, Tübingen 1999 (Bibliothek des Deutschen Historischen Instituts in Rom, 89), S. 367. Dieselbe Ungenauigkeit ist bei dem 1347 verstorbenen, aber in der Rubrik "Sec. XV « verzeichneten Bischof Omodeo Papazurrı festzustellen: EGIDI, Necrologi (wie Anm. 11), I, S. 119; zur Person vgl. ReHBERG, Kanoniker, S. 269.

${ }^{32}$ Vgl. zu ihren Stiftungen Agostino Paravicini Bagliani, I testamenti dei cardinali del Duecento, Rom 1980 (Miscellanea della Società Romana di Storia Patria, 25), S. 355, 439.

${ }^{33}$ Vgl. EGIDI, Necrologi (wie Anm. 11), I, S. 148, 150, 154, 164. Die im Archiv des Hospitals aufbewahrten Testamente können noch weitere Namen liefern. Ausnahmen von der Re- 
14. Jahrhundert, in der Zeit der Abwesenheit der Kurie, keine Päpste und Kardinäle mehr verzeichnet wurden. Die unverkennbare Nachlässigkeit im liturgischen Gebetsgedenken wird damit greifbar.

Während das Nekrologium von S. Spirito eher eine interne Angelegenheit war und sich an die eigenen Mitglieder und Wohltäter wandte, stand die Bruderschaft (fraternitas), die mit dem Orden verbunden war, allen offen, die bereit waren, einen bestimmten Jahresbeitrag zur Unterstützung seiner Krankenpflege zu leisten. Bereits die Ordensregel erwähnt einen liber fraternitatis, der aber nicht erhalten ist. Spätestens in der Avignoneser Zeit wurde die zentrale Führung von Mitgliedslisten aufgegeben. Erst mit der Wiederbelebung der Bruderschaft unter Eugen IV. im Jahre 1446 legte man wieder ein solches Verzeichnis an, den bekannten Liber fraternitatis, der 1914 von Pietro Egidi ediert wurde ${ }^{34}$. Die großzügige Ausstattung des Hospitals mit außergewöhnlichen Ablässen ${ }^{35}$ und das Vorbild des Papstes und einiger Kardinäle, die der Bruderschaft persönlich beitraten, führten dazu, daß sich sofort zahlreiche prominente Kuriale und Pilger in das Buch eintragen ließen. Ebenfalls aus ganz Europa kam der Zuspruch, als Sixtus IV. 1478 eine zweite, diesmal dauerhafte, Neubelebung betrieb, die mit dem Bestreben einher ging, die Stellung des S. Spirito in Sassia als - auch äußerlich unverkennbar - wichtigstes Krankenhaus der Stadt zu zementieren ${ }^{36}$. Wenn im Liber fraternitatis die Insassen ganzer nordalpiner Konvente aufgenommen wurden, so ist allerdings nicht von einer persönlichen Anwesenheit der Betreffenden in Rom auszugehen, sondern von einer Registrierung, die von einem Prokurator veranlaßt worden war $^{37}$. Dies ist besonders bei den Einträgen gekrönter Häupter und Fürsten zu

gel sind die Namen Francesco Hominisdei und Omodeo Papazurri (vgl. zu ihnen Anm. 31), die sehr gute Beziehungen zu den Colonna unterhalten haben.

${ }^{34}$ EGIDI, Necrologi (wie Anm. 11), II, S. 107-446.

${ }^{35}$ Vgl. Andreas REHBERG, Nuntii - questuarii - falsarii. L'ospedale di S. Spirito in Sassia e la raccolta delle elemosine nel periodo avignonese, in: Mélanges de l'École française de Rome. Moyen Âge/Temps modernes 115 (2003) S. 41-132, bes. S. 120-124.

${ }^{36} \mathrm{Vgl}$. EsPosito, Gli ospedali romani (wie Anm. 3), S. 248f. und - für die hervorragende künstlerische Ausstattung - Eunice D. HowE, The Hospital of Santo Spirito and Pope Sixtus IV, New York, London 1978 sowie den in Anm. 17 zitierten zweiteiligen Tagungsband.

${ }^{37}$ Vgl. als Beispiel die Schwestern eines Tertiarinnen-Konvents in Urach sowie die fratres der Filiale des Heilig-Geist-Ordens in Memmingen, recepti ad preces fratris Iacobi Matzenberger: EGIDI, Necrologi (wie Anm. 11), II, S. 434. Hier stellt sich das Problem, daß offenbar nicht alle, die sich bei den Entsandten des Hospitals einen Bruderschaftsbrief verschafften, auch einen Eintrag im römischen Liber fraternitatis erhielten (vgl. dazu das Eingeständnis des anonymen Schreibers des Liber in EGIDI, Necrologi (wie Anm. 11), II, S. 150). $\mathrm{Zu}$ den mitunter sehr zweifelhaften Methoden der Kollektoren von S. Spirito vgl. REHBERG, Nuntii (wie Anm. 35), S. 88, 102-119. 
erkennen $^{38}$. Der aufkommende Buchdruck trug zur größeren Verbreitung der geistlichen Anreize, die mit der Bruderschaft verbunden waren, bei. Die internationale Zusammensetzung ${ }^{39}$ unterscheidet die Heiliggeist-Bruderschaft von anderen römischen Bruderschaften, die enger mit der Stadt Rom in Verbindung standen oder sich an einer Sprachgruppe orientierten. Konkret auf unsere Ausgangsfrage bezogen, spiegelt der Liber fraternitatis die besondere Rolle des Heiliggeist-Spitals für den päpstlichen Hof wider, der nach der Rückkehr aus Avignon seinen festen Platz im Vatikan gefunden hatte und nun in S. Spirito seine natürliche medizinische Versorgungsstation besaß, die auch den Römern offenstand.

\section{Das Erlöser-Krankenhaus am Lateran (ein `Bürger -Hospital)}

Das Vorbild von S. Spirito war zu gewaltig, als daß es nicht eine Reaktion seitens der Stadt Rom und seiner Bürger hervorgerufen hätte, die die päpstliche Gründung bewunderten aber auch ihre wirtschaftliche Macht neideten. Ihre anfangs wohl noch unbewußte Antwort ${ }^{40}$ war das Hospital des SS. Salvatore (heute S. Giovanni), ein Krankenhospiz, dessen Gründung auf die Initiative einiger römischer Bürger zurückging und das nicht von Ordensangehörigen, sondern weitgehend von Laien getragen wurde. Das Ospedale di S. Giovanni ist heute das größte Hospital Roms; seine Anfänge waren dagegen bescheiden. Die Präsenz der Laterankirche und des päpstlichen Palastes als Verwaltungszentrum der römischen Kirche, die bis zum Avignoneser Exil das Ziel zahlreicher Besucher und Pilger waren, ließ schon früh karitative Einrichtungen in

${ }^{38}$ Beispiele: EGIDI, Necrologi (wie Anm. 11), II, S. 141 (König Heinrich VII. von England, König Johann von Schweden), 147 (die Herzöge von Sachsen 1478, Erzherzog Maximilian 1483).

${ }^{39}$ Die Fremden, die im Bruderschaftsbuch von S. Spirito in Sassia verzeichnet sind, sind zum Gegenstand von Einzeluntersuchungen zur Präsenz der verschiedenen nationalen Gruppen in Rom geworden. Für einige Titel zu den deutschen Namen sei verwiesen auf REH. BERG, Nuntii (wie Anm. 35), S. 123 Anm. 325.

${ }^{40}$ Hier soll nicht eine anachronistische Konfliktsituation konstruiert werden; sicher erscheint aber, daß die Gründung eines Bürgerhospizes nicht bei St. Peter sondern am gegenüberliegenden Pol der Stadt, bei der Laterankirche, zu einem Zeitpunkt, als sich der politische Schwerpunkt weg von der alten Papstresidenz hin zu der neuen am Vatikan (zumal bei der Rückkehr der Päpste) verlagerte, programmatische Bedeutung hatte. Vgl. zum urbanistischen Kontext Giovanna CURCio, L'Ospedale di S. Giovanni in Laterano: funzione urbana di una istituzione ospedaliera, in: Storia dell'arte 32 (1978) S. 23-39; 36 (1979) S. 103-130. 
ihrer Nähe entstehen ${ }^{41}$. Einige Häuser zwischen der Via Merulana und Via S. Giovanni (vormals Via Maior) sollen von Kardinal Giovanni Colonna von S. Prassede um 1216 gestiftet worden $\operatorname{sein}^{42}$. Von dieser Gründung sind die Gebäude auf der gegenüberliegenden Seite der Via Maior in Richtung SS. Quattro Coronati, die neben einer dem Erzengel Michael geweihten Kapelle (= $\mathrm{S}$. Angelo) lagen, zu unterscheiden ${ }^{43}$, die ab der 30er Jahre des 14. Jahrhunderts von der Bruderschaft der dem Bildnis Unseres Erlösers an der Sancta Sanctorum Empfohlenen (Societas raccomandatorum imaginis Salvatoris nostri ad Sancta Sanctorum) für einen Hospitalsbau erworben wurden. Noch heute liest man, daß Kardinal Giovanni sowohl das Hospital als auch die Sozietät gegründet habe; diese seien dann von Kardinal Pietro Colonna $(\dagger 1326)$ als dem eigentlichen fundator neu geordnet worden ${ }^{44}$. Die Meinung, daß Pietro der Gründer der Bruderschaft war, kann sich auf das Zeugnis der ersten Jahrgedächtnis-Liste und des Katasters berufen, die Niccolò Signorili, der Martin V. (aus dem Hause Colonna!) nahestand, um 1419 angelegt hat ${ }^{45}$. Ohne Quellenangabe spricht er davon, daß Pietro fuit pater fundator, benefactor et defensor dicte nostre societatis ${ }^{46}$. Dieses Verdienst Pietros wird auch durch den Wunsch des Kanonikers von S. Maria Maggiore Stefano Vaschi erhärtet, der 1398 in seinem Testament verfügte, daß die Mitglieder der Bruderschaft sein Jahrgedächtnis so gestalten sollten, wie sie faciunt in dicta ecclesia sancte Marie Maioris pro anima condam reverendissimi

${ }^{41} \mathrm{Zu}$ den bereits in Lateran-Nähe bestehenden Hospizen vgl. BiRCH, Pilgrimage to Rome (wie Anm. 7), S. 137-140.

${ }^{42}$ CANEZZA, Gli arcispedali (wie Anm. 7), S. 183f. Vgl. zur vermeintlichen Gründung des Kardinals von S. Prassede Georges RohaULT DE FleURY, Le Latran au Moyen Ảge, Paris 1877, S. 164 (mit Datierung in das Jahr 1226). Zurückzuweisen sind die unfundierten elfseitigen Angaben bei Antonio PALICA, Origine e successivo sviluppo dell'Ospedale del SS.mo Salvatore in Laterano. Appunti storici, Rom 1892, besonders S. 3f. Unklar ist die Meinung, wonach die Gründung der Colonna mit einer angeblich 1289 erfolgten Auflösung eines Hospitals der Antoniter in Verbindung steht: EsPosito, Gli ospedali romani (wie Anm. 3). S. 236. Richtig gibt den Sachverhalt, der sich auf das Hospital S. Andrea de Piscina bei S. Maria Maggiore bezieht (s. Anm. 56), CANEZZA, Gli arcispedali (wie Anm. 7), S. 180 wieder; die Quelle ist Registres de Nicolas IV, ed. Emest LANGLOIS, Paris 1898-1938 (Bibliothèque des Écoles françaises d'Athènes et de Rome 2. ser.), Nr. 1997 (1289 Dez. 23).

${ }^{43}$ CANEZZA, Gli arcispedali (wie Anm. 7), S. 185.

${ }^{44}$ Vgl. ibid. S. 187 (mit der älteren Literatur). Diese auf reiner Spekulation basierende Meinung hat bei CURCIO, L'Ospedale (wie Anm. 40), S. 26 Anklang gefunden.

${ }^{45}$ EgIDI, Necrologi (wie Anm. 11), I, S. 311; DYKMANS, Les obituaires (wie Anm. 23), S. 629. Eine genaue Untersuchung nach kodikologischen und paläographischen Gesichtspunkten steht noch aus.

${ }^{46}$ EGIDI, Necrologi (wie Anm. 11), I, S. 317 (hier Zitat). Im Kataster (bzw. Libro dei fratelli) ist von anderer Hand ebenfalls von Pietro als dem fundator huius quidem Sotietatis die Rede. DERS., Necrologi (wie Anm. 11), II, S. 455. 
in Christo patris et domini domini Petri de Columpna dudum sancte romane ecclesie cardinalis ${ }^{47}$.

Wann gründete der Kardinal die Bruderschaft? Das Gründungsjahr schwankt in der Literatur meist zwischen 1288 und 1318. Sicher, 1288 war Pietro Kardinal geworden ${ }^{48}$; aber erst 1318 wird die Bruderschaft in den Quellen erwähnt, als Johannes XXII. auf ihre Bitte einen Ablaß für die Besucher des Salvator-Ikone in der dem hl. Laurentius geweihten Kirche (wegen ihrer Reliquienschätze Sancta Sanctorum genannt) gewährte ${ }^{49}$. Die Mitwirkung der Colonna am Erwerb des Ablaßbriefes geht indirekt dadurch hervor, daß die Sigle „P. Set." des Schreibers der Bulle mit großer Wahrscheinlichkeit auf einen Familiaren des Kardinal Pietro Colonna namens Petrus de Setia (Pietro aus Sezze) verweist, der gleichzeitig päpstlicher scriptor war (eine nicht seltene Ämter-Kombination!) $)^{50}$. Die ersten Statuten der Gemeinschaft von 1331 nehmen allerdings weder auf den fünf Jahre zuvor verstorbenen Kardinal noch auf ein Hospital Bezug ${ }^{51}$. Es gibt also keinen direkten Beleg für die Pietro zugeschriebene Ehre. Und doch darf man seinen Namen nicht von vorneherein verwerfen. Wenn er die Bruderschaft wahrscheinlich auch nicht persönlich gegründet hat, so muß sie doch von Anfang an unter dem Patronat des Colonna gestanden haben. Dafür gibt es nun mehrere Indizien, wenn man die Kirchenpfründen der Colonna und die Zusammensetzung ihrer Klientel betrachtet. Der Kardinal war nämlich seit 1307 Kommendatar von S. Lorenzo ad Sancta Sanctorum, wo das verehrte Erlöserbild aufbewahrt wurde ${ }^{52}$. Außerdem war er von 1306 bis zu seinem Tode 1326 Erzpriester der benachbarten Laterankirche, der die Laurentius-Kirche und damit auch deren Reliquienschatz unterstanden. Dank der im Laterankapitel sitzenden Kanoniker aus der eigenen Familie oder ihrer Klientel war der Einfluß der Colonna dort selbst im Intervall von 1326 bis 1342 recht groß, als der Archipresbyterat nicht von ih-

\footnotetext{
${ }^{47}$ Biblioteca Apostolica Vaticana (hinfort BAV), Archivio del capitolo di S. Maria Maggiore, cart. 71, n. 163 (1398 Juli 17-19).

${ }^{48}$ Für 1288 spricht sich u.a. CURCIO, L'Ospedale (wie Anm. 40), S. 26 aus. Auch Matizia MARONI LUMBROSO, Antonio MARTINI, Le confraternite romane nelle loro chiese, Rom 1963, S. 394 399, hier S. 394f., favorisiert Pietro Colonna als Gründer der Bruderschaft.

${ }^{49}$ Lettres communes. Jean XXII (1316-1334), ed. Guillaume MOLLAT, Paris 1904-1947 (Bibliothèque des Écoles françaises d'Athènes et de Rome, $3^{\mathrm{c}}$ série $\mathrm{I}^{\mathrm{bis}}$ ), $\mathrm{Nr} .8347$.

${ }^{50}$ Die Schreiber-Sigle findet sich nur in einer modernen Abschrift des Dokuments (das Original ist verloren): ASR, Ospedale di S. Spirito, 1408 (nicht paginiert). Eine weitere Kopie ibid., 1005, fol. 3r existiert nicht mehr (die ersten 13 folia sind herausgetrennt worden). Zu dem genannten Pietro di Sezze vgl. Andreas ReHBERG, Kirche und Macht im römischen Trecento. Die Colonna und ibre Klientel auf dem kurialen Pfründenmarkt (1278-1378), Tübingen 1999 (Bibliothek des Deutschen Historischen Instituts in Rom, 88), S. 472 (P 70).

${ }^{51}$ Ediert in PaVAN, Gli statuti (wie Anm. 5), S. 62-68.

52 Regestum Clementis Papae V ex vaticanis archetypis ..., cura et studio monachorum ordinis S. Benedicti, Rom 1884-1957, Nr. 2007 (1307 Nov. 8).
} 
nen besetzt wurde. Damals, genauer im Jahre 1336, erwarben die Guardiane der Bruderschaft Giovanni di Nardo und Egidio di Andrea Franchi vom Laterankapitel einen Garten. Sechs Kanoniker, darunter drei erklärte ColonnaFreunde, nahmen damals an der Sitzung des Kapitels teil und genehmigten den Vertrag $^{53} .1348$ standen dann die Bauarbeiten am Hospital S. Angelo vor dem Abschluß, als der Guardian Francesco Vecchi ${ }^{54}$ ein angrenzendes palatium von der Familie Novelli kaufte. Unter den vier Laterankanonikern, die damals offenbar noch im pestverseuchten, heißen Rom ausharten und am 3. August 1348 ihre Zustimmung zu dem Besitzwechsel gaben, waren ein Colonna und ein Familiar des einen Monat zuvor verstorbenen Kardinals Giovanni Colon$\mathrm{na}^{55}$. Als Fazit kann man zwar weitgehend ausschließen, daß die Colonna die eigentlichen Gründer des Erlöser-Hospitals gewesen waren; man wird aber ihre Rolle bei seinen Anfängen nicht unterschätzen dürfen. Die Colonna hatten zudem ein politisches Interesse daran, dem von ihren Rivalen, den Orsini, dominierten Hospital von S. Spirito ein >eigenes entgegenzusetzen ${ }^{56}$.

${ }^{53}$ Im einzelnen waren dies Giacomo di Riccardo Annibaldi, Cecco di Pietro Annibaldi, Giovanni Foschi de Berta, Pietro Alexii, Angelo di Giacomo Pecorari e Francesco Guidonis: REHBERG, Kanoniker (wie Anm. 31), S. 212. Zu den genannten Kanonikern vgl. ibid. Nr. L $4,5,61,63,64,127$.

${ }^{54}$ Der Notar Francesco Vecchi war eine bekannte Figur der römischen Stadtpolitik und 1354 am Sturz Cola di Rienzos, der besonders die Familie Colonna herausgefordert hatte, beteiligt. Er soll dem Tribunen angeblich auch den ersten Todesstoß versetzt haben: ANONIMO ROMANO, Cronica, edizione critica a cura di Giuseppe PORTA, Mailand 1979, S. 264 (allora Cecco dello Viecchio impuinao mano a uno stuocco e deoli nello ventre). Während Pietro EGIDI, Chi era l'uccisore di Cola di Rienzo, in: Miscellanea per le Nozze CrocioniRuscelloni, Rom 1908, davon ausgeht, daß der Mörder ein Anhänger der Colonna war, sieht in ihm Armanda Collins, Greater than Emperor. Cola di Rienzo (ca. 1313-1354) and the World of Fourteenth Century Rome, Ann Arbor 2002, S. 232-235 nur einen Popolanen, der mit seiner Tat Colas nobsessive, personal war with the Colonna« beenden und wa new popular regime « schmieden wollte (S. 235: Zitate). Die Amnestie der Mörder Colas wurde 1355 an der Kurie von einer parteiübergreifenden Gesandtschaft aus Rom betrieben, deren promınentestes Mitglied immerhin Stefano Colonna, Propst von Saint-Omer, war: REHBERG, Kirche und Macht (wie Anm. 50), S. 371 f.

${ }^{5 s}$ Die vier Kanoniker waren Giacomo Annibaldi (auch als Jacobus de Cavis bezeichnet), Pietro de Scantrilgia, Bertoldo Marrancii und Pietro Camillo Colonna: CANEZZA, Gli arcispedali (wie Anm. 7), S. 187f.; REHBERG, Kanoniker (wie Anm. 31), S. 213. Zu den genannten Kanonikern vgl. ibid. Nr. L 5, 38, 95, 122.

${ }^{56}$ Die Einwirkung der römischen Baronalfamilien auf das Gesundheitswesen in Rom ist auch sonst nachzuweisen. So war S. Giacomo in Augusta ebenfalls eine Gründung der Colonna: CAnEzZA, Gli arcispedali (wie Anm. 7), S. 203ff.; Renzo U. MONTINI, S. Giacomo in Augusta, Le chiese di Roma illustrate, Rom o. J. [1957-1959], S. 4ff. Der Kardinal Pietro Capocci $(\dagger 1259)$ verfügte testamentarisch den Bau des Hospitals S. Andrea (de Piscinula) neben der Basilika S. Maria Maggiore (s. oben Anm. 42), das von einem Antoniter geleitet wurde: Agostino ParaviCini Bagliani, Capocci, Pietro, in: Dizionario biografico degli Italiani, Bd. 18, Rom 1975, S. 604-608, hier S. 607. 
Wenden wir uns den Raccomandati selbst zu. Unbestritten ist, daß sie schon bald mehr als nur eine Gruppe frommer Bürger waren, die sich, dem Auftrag ihrer Statuten gemäß, dem Gebet und guten Werken widmeten ${ }^{57}$. Daß sie von Anfang an öffentliche Wirkung entfalteten, lag im Kult des Erlöserbildes selbst begründet. Diese Ikone wurde an Mariä Himmelfahrt in einer vom römischen Magistrat begleiteten Prozession unter allgemeiner Anteilnahme und großem Aufwand von ihrer Kirche beim Lateran - symbolträchtig in einem weiten Bogen am Kolosseum und dem Kapitol vorbei - nach S. Maria Maggiore getragen und dort seiner >Mutter, einer ebenfalls hochverehrten Ikone, vorgestellt. Durch die Jahrhunderte war dieser Brauch zu einer wichtigen Manifestation des Selbstbewußtseins der römischen Stadtkommune geworden, die päpstlicherseits nicht immer mit Wohlwollen betrachtet wurde ${ }^{58}$. Kein Wunder also, daß die Colonna, die an den beiden Basiliken des Anfangs- und Endpunktes der Prozession eine bedeutende Rolle spielten ${ }^{59}$, an den Aktivitäten der Fraternität Anteil nahmen.

Was kann aber schon jetzt hinsichtlich der sozialen Zusammensetzung der Salvatorbruderschaft ausgesagt werden? Dazu ein kurzes Wort zur Entstehung des sog. Liber anniversariorum ${ }^{60}$ und der Mitgliedsliste (Libro dei fratelli) der Salvatorbruderschaft ${ }^{61}$, die beide von Niccolò Signorili in Angriff genommen wurden, nachdem offenbar bereits viel Quellenmaterial, auf das er sich ansonsten, soweit möglich, stützte, verloren war. So führt Signorili seine Güter- und Mitgliederlisten kaum in die Zeit vor das Pestjahr 1348 zurück, und auch für

${ }^{57}$ Paola PAVAN, La confraternita del Salvatore nella società romana del Tre-Quattrocento, in: Luigi FIORANI (Hg.), Le confraternite romane. Esperienza religiosa, società, committenza artistica (Colloquio della Fondazione Caetani, Roma 14-15 maggio 1982), in: Ricerche per la storia religiosa di Roma 5 (1984) S. 81-90, hier S. 85ff.

${ }^{58}$ Diesen komplexen Bezügen ist Gerhard WOLF, Salus populi romani. Die Geschichte römischer Kultbilder im Mittelalter, Weinheim 1990 nachgegangen.

${ }^{59}$ Die Colonna waren nicht nur an S. Giovanni, sondern auch an S. Maria Maggiore lange Zeit Erzpriester gewesen und hatten dort ebenfalls zahlreiche Kanoniker gestellt: REHBERG, Kanoniker (wie Anm. 31), S. 112-120, 128-131.

${ }^{60}$ Ausgangspunkt ist die Edition in EGIDI, Necrologi (wie Anm. 11), I, S. 311-541, die leider auf einer Kopie des 17. Jahrhunderts beruht (ASR, Ospedale SS. Salvatore, catasto, n. 2). Der Herausgeber mußte sechs Jahre später eingestehen, daß das verloren geglaubte Original von der Hand des Signorili (ibid. n. 1) noch existierte: EGIDI, Necrologi (wie Anm. 11), II, S. 447. Zusammen mit dem Libro dei fratelli müßten all diese Texte auch mit späteren Kompilationen abgeglichen werden: ibid. n. 1009 (Liber societatis aus dem Jahr 1462, mit der Anordnung der Jahresgedächtnisse nach Kirchen) und 1008 (Libro degli anniversari von 1461). Egidi selbst hat an anderer Stelle ein ebenfalls von 1461 stammendes Verzeichnis der Anniversarien, diesmal in Volgare verfaßt (ibid., catasti, n. 25), ediert: DERS., Libro di anniversari in volgare dell'Ospedale del Salvatore, in: Archivio della Società Romana di Storia Patria 31 (1908) S. 169-209. Eine Neubearbeitung des Materials könnte die Chancen einer on-line-Edition nutzen (vgl. Anm. 12).

${ }^{61}$ Es handelt sich um ein Original: EGIDI, Necrologi (wie Anm. 11), II, S. 447-531. 
die Jahrzehnte danach ist die Überlieferung noch fragmentarisch, so daß er selbst mitunter auf die Aussagen von älteren Mitgliedern zurückgreifen muß$\mathrm{te}^{62}$. Da der Urkundenbestand des Archivio dell'Ospedale SS. Salvatore bis auf die rekonstruierbaren Verluste in jüngster Zeit weitgehend derselbe ist, den schon Signorili auswerten konnte, ist man für die Zeit vor 1348 auf wenjge Urkunden beschränkt ${ }^{63}$. Hier hilft nun die prosopographische Erfassung der Namen der bekannten Mitglieder der Bruderschaft und derjenigen, die von ihr Jahresgedächtnisse feiern ließen, weiter. Eine Studie über das gesamte Material käme angesichts der enormen Bedeutung der Bruderschaft einer Analyse nahezu der gesamten gehobenen Mittel- und Oberschicht Roms im Spätmittelalter und darüber hinaus gleich. Für die Zwecke unseres Beitrags ist es ausreichend, sich auf die ältesten Einträge, die in die Zeit um 1350-1370 gehören, zu beschränken. Es ist unverkennbar, daß einige dieser Namen mit dem Colonna-Ambiente verknüpft sind ${ }^{64}$. Ein Vergleich hat ergeben, daß von den 72 nicht-baronalen Familien, die vor 1348 auf dem kurialen Pfründenmarkt in Kontakt mit den Colonna standen, 33 - also mit $46 \%$ fast die Hälfte - im Laufe des Trecento und in den ersten Jahrzehnten des Quattrocento nachweisbar Verbindungen zur Salvatorbruderschaft hatten ${ }^{65}$. Auch wenn diese Zahlen angesichts der lückenhaften Überlieferung nur einen ersten, fragmentarischen Eindruck vermitteln und die Belege aus späterer Zeit keineswegs überbewertet werden sollen, ist die starke Präsenz Colonna-naher Familien doch deutlich. Dabei übertrifft die Zahl der Geschlechter aus der alten Aristokratie die der Familien, die der Mittelschicht zuzuordnen sind (20:13).

Die Mitgliedschaft etlicher Colonna ist ab der zweiten Hälfte des 14. Jahrhunderts belegt ${ }^{66}$. Der Laterankanoniker Pietro-Camillo und Matteo di Gior-

${ }^{62} \mathrm{Zu}$ den Anstrengungen Signorilis vgl. ibid. I, S. 311f., 331 (Berufung auf mündliche Aussagen antiquorum confratrum nostrorum).

${ }^{63}$ Einen ersten Überblick über die Situation des Bruderschaftsarchiv bietet Anna ESPOSITO, SS. Salvatore ad Sancta Sanctorum, in: Luigi FroRANI (Hg.), Storiografia e archivi delle confraternite romane, in: Ricerche per la storia religiosa di Roma 6 (1985) S. 390-392.

${ }^{64}$ Vgl. EGIDI, Necrologi (wie Anm. 11), I, S. 317-319. mit Namen wie Pietro spetiarius (ein Ognisanti-Mancini), Stefano Tosetti (aber auch für seinen nicht verzeichneten Verwandten Lello, den ,Laelius( Petrarcas, war ein Anniversar vorgesehen worden!), Simone Malabranca, Giovanni Mardoni und Giannotto Boboni. Zur Identifizierung und sozialen Verortung dieser Persönlichkeiten siehe Andreas ReHBERG, Familien aus Rom und die Colonna auf dem kurialen Pfründenmarkt (1278-1378), 2 Teile, in: Quellen und Forschungen aus italienischen Archiven und Bibliotheken 78 (1998) S. 1-122, 79 (1999) S. 99-214, hier I, 36, 56, 91; II, 133, 135, 186.

${ }^{65}$ Hinweise auf Mitgliedschaft und sonstige Kontakte (z.B. Jahrgedächtnisse) zu ihr finden sich ibid., unter den Familien-Siglen F 9, 10f., 13, 16, 17f., 21-24, 26, 28, 32, 36, 41 f., 44 46, die zur alten Aristokratic zählen, und in den popularen Familien F 50f., 53, 57f., 61, 64f., 69-72, 79. Zur Präsenz sonstiger Barone in den Listen der Mitglieder und Stifter vgl, unten Anm. 73.

${ }^{66}$ EGIDI, Necrologi (wie Anm. 11), II, S. 468. 
dano ließen ihre Seelenmessen von ihr ausrichten, wobei letzterer ihr dafür ein Viertel des Kolosseums schenkte ${ }^{67}$. Filippa Tedallini, die Frau des verstorbenen Bartolomeo Fucii ex dominis de Gallicano de Columpna, schenkte im April 1394 der Salvatorbruderschaft für eine Meßstiftung in S. Silvestro in Capite ein Haus im Rione Campitelli ${ }^{68}$. Es war gewiß kein Zufall, daß im Monat darauf Maria, die Frau des Landolfo Colonna von Riofreddo, den Jacobus Johannis Andree, der damals Guardian der Bruderschaft war, zum Schiedsrichter eines Streits zwischen ihr und Symodea Orsini bestellte ${ }^{69}$.

Schon diese ersten Ergebnisse zu den Klientelbeziehungen und die Überprüfung des sozialen Hintergrundes der ersten faßbaren Mitglieder und Wohltäter relativieren die Vorstellung, daß sie vorwiegend mercanti gewesen waren und von einem Antagonismus $>$ Bürger gegen Adel< beseelt waren ${ }^{70}$. Es zeigt sich, daB man sozial und zeitlich mehr differenzieren und vor allem die nahezu alle Lebensreiche berührenden, aber vielfach unterschätzten Gegensätze zwischen den wohlbekannten Adelsparteien berücksichtigen $m u B^{71}$. Die von einer tiefen politischen Krise erfaßten Colonna dürften allerdings schon um die Mitte des 14. Jahrhunderts ihren unmittelbaren Einfluß auf die Bruderschaft verloren

${ }^{67}$ Ibid. I, S. 321.

${ }^{68}$ BAV, Arch. S. Angelo in Pescheria, V17, not. Antonio Scambii, f. 20rf. (1394 Apr. 2); EGIDI, Necrologi (wie Anm. 11), I, S. 326 gibt aber als Ort der Meßstiftung win eccl. S. Ioannis de Mercato, in capella S. Annae« an; vgl. Giuseppe TOMASSETTI, La Campagna Romana, antica, medioevale e moderna, hg. von Luisa CHIUMENTI, Fernando BILANCIA. 7 Bde., Florenz ${ }^{2}$ 1979-1980 (Arte e archeologia. Studi e documenti, 12-18), hier ll1, S. 598.

${ }^{69}$ BAV, Arch. S. Angelo in Pescheria, I/17, not. Antonio Scambii, fol. $21 \mathrm{r}$ (1394 Apr. 10). Der Streit ging um sequestrierte Schweine. Das Bruderschaftsamt des Vermittlers geht hervor aus ibid. fol. 20r.

${ }^{70}$ Typisch für diese Sicht ist CuRCIo, L'ospedale (wie Anm. 40), S. 17ff. Vgl. auch PavaN. Gli statuti (wie Anm. 5), S. 37f. und DiES., La confraternita (wie Anm. 57), S. 83.

${ }^{71}$ Die römische Stadtgeschichtsschreibung hat erst jüngst damit begonnen, die Parteıgegensätze in Rom in Beziehung zu den sozialen Gruppen zu setzen: vgl. REHBERG, Kirche und Macht (wie Anm. 50), S. 242-306; DERS., Clientele e fazioni nell'azione politica di Cola di Rienzo = DERS., Anna MODIGlianI, Cola di Rienzo e il comune di Roma, I (RR inedita, 33,1), Rom 2004. Wenn Pavan, La confratemita del Salvatore (wie Anm. 57), S. 83f.. die "famiglie di recenti se non recentissime fortune « vom Schlage der Maddaleni, Porcari, Gibelli und Valentini in einem Atemzug mit alten Stadtadelsfamilien wie Galgani, Ilperini, Capogalli, Rossi, Foschi da Berta und Sordi nennt, werden die Unterschiede zwischen den beiden Gruppen zu stark relativiert, zumal sie politisch keineswegs an einem Strang zogen. Von den Familien der zweiten Gruppe standen die vier zuletzt genannten Geschlechter traditionell den Colonna nahe: REHBERG, Familien (wie Anm. 64), I, F 17; II, F 36, 41, 58. Vgl. zur gesellschaftlichen und politischen Entwicklung Roms im Hoch- und Spätmittelalter Jean Claude MAIRE VigueUR, Il Comune Romano, in: André Vauchez (Hg.), Roma medievale (VII-XIV secolo), Rom, Bari 2001 (Storia di Roma dall'antichità a oggi, 2), S. 117-157; Sandro CAROCCI, Marco VENDITTELLI, Societż ed economia (1050-1420), in: ibid. S. 71116. Unersetzt ist weiterhin Eugenio DUPRÉ THESEIDER, Roma dal comune di popolo alla signoria pontificia (1252-1377), Bologna 1952 (Storia di Roma, 11). 
haben. Ohne daß man eine Verbindung zu den Aktionen des bekannten Tribunen Cola di Rienzo ( $\dagger$ 1354) erkennen kann ${ }^{72}$, wurde die Salvator-Sozietät immer mehr zu einem Sammelbecken der neuen kommunalen Führungsgruppe. Die wirtschaftlichen Aufsteiger drängten aber offenbar verstärkt erst im letzten Viertel des Trecento in die Bruderschaft. Damals kamen viele Mitglieder auch aus Stadtteilen, die von den Orsini dominiert wurden. Ja, selbst die Orsini und andere Baronalfamilien waren jetzt mit Familienmitgliedern und Exponenten ihrer Klientel vertreten ${ }^{73}$. Dieser Wandel läßt sich mit dem Erfolg der Bruderschaft erklären, der neue Kreise anzog. Den Raccomandati wuchsen sogar öffentliche Ordnungsfunktionen zu, deren wichtigste die Jurisdiktion im Bereich zwischen dem Kolosseum und dem Lateran war, die ihnen die römische Kommune 1386 überlie $\beta^{74}$. Von den alten Beziehungen zu den Colonna kündete bald nur noch das stets gepflegte Andenken des Gründers und die ungebrochene Präsenz einiger Familien in den Listen der Mitglieder und Stifter. Noch um 1400 waren auffallig viele Exponenten der Colonna-nahen Partei der Nobili in der Salvatorbruderschaft engagiert ${ }^{75}$. Unter Martin V. Colonna, der schon als Kardinal Mitglied war, erreichte die Verflechtung der Colonna und ihrer Klientel mit der Sozietät einen neuen, wenngleich nur kurzen Höhepunkt $^{76}$.

Für eine weitere Beschäftigung mit der sozialen Zusammensetzung der Salvatorbruderschaft im 15. und frühen 16. Jahrhundert muß man sich noch einmal die Struktur der beiden Hauptquellen vergegenwärtigen. Der Libro dei fratelli ordnet Kardinäle, Prälaten, Priester, Barone sowie die nach Stadteilen (Rioni) unterteilten sonstigen Laien in eigene Rubriken, die von den verschiedenen Kompilatoren nach Signorili weitergeführt wurden ${ }^{77}$. Frauen sind im

${ }^{72}$ Cola di Rienzo, dessen auch persönlich gefärbte Feindschaft zu den Colonna bekannt ist, nahm nie auf die Salvator-Bruderschaft Bezug und förderte sie und ihr noch junges Hospital - im Gegensatz zu S. Spirito - nicht weiter. Zu einem seiner Mörder, der offenbar Guardian der Erlöser-Bruderschaft war, vgl. oben Anm. 54.

${ }^{73}$ Vgl. EGIDI, Necrologi (wie Anm. 11), I, S. 324 (Iacobellus de Ursinis; zu dessen Tochter Giovanna vgl. S. 327), S. 328 (die Orsini-Witwe Caterina von Supino). Zu späteren Beitritten von Orsini, aber auch von Capocci, Normanni und Savelli, sowie zum Mitglied Onorato Caetani ( $\uparrow 1400$ ) vgl. EGIDI, Necrologi (wie Anm. 11), II, S. 468.

${ }^{74} \mathrm{Vgl}$. Pasquale ADINOLFI, Laterano e Via Maggiore. Saggio della topografia di Roma nell'età di mezzo, Rom 1857, S. 93f., 140-149; CURCIO, L'ospedale (wie Anm. 40), S. 27f.; PaVAN, Gli Statuti (wie Anm. 5), S. 43.

${ }^{75}$ Arnold ESCH, Bonifaz IX. und der Kirchenstaat, Tübingen 1969 (Bibliothek des Deutschen Historischen Instituts in Rom, 29), S. 244.

${ }^{76} \mathrm{Vgl}$. Andreas REHBERG, Etsi prudens paterfamilias ... pro pace suorum sapienter providet. Le ripercussioni del nepotismo di Martino V a Roma e nel Lazio, in: Miriam CHIABÒ u.a. (Hg.), Alle origini della nuova Roma. Martino V (1417-1431), Atti del convegno, Roma 2-5 marzo 1992, Rom 1992 (Nuovi studi storici, 20), S. 225-282, hier S. 239 und S. 263, Anm. 181.

77 Siehe EgIDI, Necrologi (wie Anm. 11), II, S. 456-469, 505-507. Für die nicht-baronalen Laien vgl. ibid., II, S. 469-504 und 507-529. 
Liber anniversariorum stark präsent ${ }^{78}$, als Mitglieder werden sie aber im Libro dei fratelli erst ab dem Jubiläumsjahr 1500 verzeichnet ${ }^{79}$. Die Mitgliedschaft ging - wie auch die Statuten festlegen ${ }^{80}$ - vom verstorbenen Vater auf den Sohn über ${ }^{81}$. Wichtig wurde, daß sich unter Martin V. die Salvatorbruderschaft, die aus Angst vor einer überhandnehmenden Klerikalisierung wiederholt den Anteil der Geistlichen auf 40 Mitglieder einzudämmen suchte (anfangs war das Verhältnis der Priester zu den Laien mit 28:72 festgelegt), auch verstärkt gegenüber dem kurialen Umfeld öffnete. Mächtige Kirchenfürsten wie Kardinal Ascanio Maria Sforza (1455-1505) sowie Barone wie der reiche Everso dell'Anguillara gehörten ihr an und vermachten ihrem Hospital großzügige Schenkungen ${ }^{82}$. Die Kurialen, die nicht aus Rom stammten, konnten sich über die Mitgliedschaft einen exklusiven Zugang zu den führenden Schichten der Stadt verschaffen. Etlichen der hier genannten Konsistorialadvokaten, Protonotaren etc. gelang es, ihre Familien in Rom zu verankern (genannt seien nur die Del Drago, die Spiriti - beide Familien stammten aus Viterbo -, die Pamphilj aus Gubbio ${ }^{83}$ und die Doria aus Genua ${ }^{84}$ ). Ende des 15. Jahrhunderts schwächte sich auch das Konkurrenz-Verhältnis zwischen S. Spirito und S. Salvatore ab. Die Beziehungen verbesserten sich spürbar, was man daran erkennen kann, daß sogar zwei Präzeptoren des Heiliggeistspitals - Pius und Constantius (sie standen von 1484 bis 1495 ihrem Krankenhaus vor) - der Salvatorbruderschaft beitreten konnten und daß der Präzeptor

${ }^{78}$ Man vgl. nur ibid., I, S. 499. Zur Rolle der Frauen in einer römischen Bruderschaft - hier der des deutschen Friedhofs - vgl. SCHulz, Confraternitas Campi Sancti (wie Anm. 5), S. 77-86. Ibid. S. 80 gibt zu bedenken, $\gg d a ß$ nicht jede römische Bruderschaft auch Platz für Schwestern hatte« (vgl. auch ibid. S. 38). Ein Anziehungspunkt waren die sogenannten Dotenstiftungen: ibid. S. 82f.; Anna ESPOSITO, Le Confraternite del matrimonio. Carità, devozione e bisogni sociali a Roma nel tardo Quattrocento (Con l'edizione degli Statuti vecchi della Compagnia della SS. Annunziata), in: Laura FORTINI (Hg.), Un'idea di Roma. Società, arte e cultura tra Umanesimo e Rinascimento, Rom 1993, S. 7-51 (englische Version in: Renaissance and Reformation, n.s. 18 [1994] S. 5-18).

${ }^{79}$ EGIDI, Necrologi (wie Anm. 11), II, S. $530 \mathrm{f}$.

${ }^{80}$ PaVAN, Gli statuti (wie Anm. 5), S. 66, 77.

${ }^{81}$ Beispiele aus den Familien Bonadies, Rustici und Mantaco: EGIDI, Necrologi (wie Anm. 11), II, S. 505, 524.

${ }^{82}$ Hier sei nur kurz auf den Kardinal Sforza eingegangen: ibid. S. 313, S. 505. Er schenkte dem Hospital zwei Casali: Marco PellegriNI, Ricerche sul patrimonio feudale e beneficiario del cardinale Ascanio Sforza, in: Archivio Storico Lombardo 122 (1996) S. 41-83, hier S. 77. Zum besagten Zugangslimit für Kleriker vgl. PAVAN, Gli statuti (wie Anm. 5), S. 63, 88, 93 (es handelt sich zuletzt um Statuten-Zusätze von 1474 und 1480).

${ }^{83} \mathrm{Zu}$ einer Bentivoglio, verwitwete Pamphilj, und ihrem Jahrtagsamt: EGIDI. Necrologi (w'L Anm. 11), I, S. 488.

${ }^{84}$ Ein Kleriker aus dem Hause Doria (de Auria) ist außerhalb seiner Rubrik verzeichnet: ibid., II, S. 525. Ein weiteres Beispiel ibid. S. 496. 
Innocenzo de Flaviis (im Amt von 1473 bis 1484) sich ein Jahrtagsamt durch sie ausrichten lie ${ }^{85}$.

Welche Funktion hatte nun das Hospital am Lateran und seine Dependancen in den Vorstellungen der Mitglieder und Wohltäter der Bruderschaft? Diese wohlhabenden Damen und Herren waren mit den Bedürfnissen der Kranken, die man damals nur als bedrückend empfinden konnte, vertraut, waren sich aber auch bewußt, der vornehmsten Laienorganisation der Stadt überhaupt anzugehören. Gaben sie kein Geld, so hinterließen sie doch Kleidungsstücke oder Bettzeug und Handtücher oder eine Bettstatt, die direkt im Krankenhaus zum Einsatz kamen ${ }^{86}$. Auch die Schenkung von Naturalien - insbesondere Wein und Getreide - waren gern gesehen ${ }^{87}$. Wichtig war, daß man insgesamt auf eine Summe von $50 \mathrm{fl}$. für die Jahrtagsstiftung $\mathrm{kam}^{88}$. Außerdem kannte S. Salvatore die Einrichtung der Oblaten, die sich gegen Überlassung ihres Eigentums bis zu ihrem Tode ein Nutzungsrecht sicherten ${ }^{89}$. Zugewanderte Ausländer ließen gelegentlich ebenfalls ihre Jahrtagsämter von der Salvatorbruderschaft austragen ${ }^{90}$. Man darf ja nicht vergessen, daß das Hospital nicht nur Einheimische, sondern auch - obgleich dies die Statuten nicht ausdrücklich vorschreiben - Pilger aufnahm ${ }^{91}$.

Es ist hier nicht der Ort, die Entwicklung des Hospitals im 15. Jahrhundert zu vertiefen ${ }^{92}$. Doch sei darauf hingewiesen, daß ihm die Päpste seit Bonifaz IX. und vor allem seit dem Römer Martin V. mehr Aufmerksamkeit widmeten $^{93}$. Dies machte das Hospital - wie gesehen - auch für Kurienmitglieder bis hin zu Kardinälen interessant. Es erlebte eine erste wirtschaftliche Blüte und war nun fest im sozialen und religiösen Bewußtsein der Römer verankert.

Damit können wir zu zwei weiteren Beispielen von Hospitälern übergehen, denen zwar keine so glänzende Zukunft beschieden war wie den Krankenhäu-

${ }^{85}$ Ibid., I, S. 529; Il, S. 505. Zu den genannten Präzeptoren vgl. DE ANGELIS, L'ospedale di S. Spirito in Saxia (wie Anm. 17), II, S. 530-533.

${ }^{86}$ EGIDI, Necrologi (wie Anm. 11), I, S. 331f., 359, 361, 395, 480 usw.

${ }^{87}$ Vgl. die Weinlieferungen ibid. S. 386, 459.

${ }^{88}$ Ein solches Rechenbeispiel bietet ibid. S. 459.

${ }^{89} \mathrm{Zu}$ zwei Beispielen sei auf ibid. S. 435, 472 hingewiesen.

90 Johanna aus Köln sorgte für ein Jahrtagsamt bezeichnenderweise in der Anima-Kirche: ibid. S. 445. Der aus Ungam stammende Pönitentiar und Dominikaner Johannes ( $\dagger$ 1364) sah es in S. Maria sopra Minerva vor: ibid. S. 323; diese Angabe ergänzt Andreas REHBERG, Die Pönitentiare in Urbe während der Avignoneser Zeit. Eine prosopographische Skizze, in: Kirsi SAlONEN, Christian KRÖTZL (Hg.), The Roman Curia, the Apostolic Penitentiary, and the Partes in the Later Middle Ages, Rom 2003 (Acta Instituti Romani Finlandiae, 28), S. 67-114, hier S. 102f. Nr. 23.

${ }_{91}$ PAVAN, La confraternita del Salvatore (wie Anm. 57), S. 85.

${ }_{92} \mathrm{Vgl}$. die Hinweise in ESPOSITO, Gli ospedali romani (wie Anm. 3), S. 239.

93 Allerdings steht noch eine systematische Erfassung der päpstlichen Privilegien für das Salvator-Hospital aus. Vgl. zu diesem Defizit auch unten Anm. 152. 
sern von S. Spirito und S. Giovanni, die aber zwei interessante Zeugen karitativen Einsatzes im spätmittelalterlichen Rom darstellen.

\section{Das Hospital von S. Maria in Portico (Spital einer kleineren Bruderschaft)}

Die societas Sancte Marie de Porticu bzw. Compagnia di S. Maria in Portico wurde im 15. Jahrhundert zur Unterstützung des Hospitals an der gleichnamigen Kirche (im Seicento durch den Neubau S. Maria in Campitelli ersetzt) und zur Verehrung eines dort aufbewahrten wundertätigen Muttergottesbildes gegründet. 1505 schloß sich die Bruderschaft mit der Confraternita di S. Maria delle Grazie und derjenigen della Consolazione zum Sodalizio di S. Maria sde vita aeternar zusammen, der sich vor allem um das Hospital sdella Consolazioner am Fuß des Kapitols (zum Forum hin) kümmerte und deshalb auch bald dessen Name (della Consolazione) übernahm ${ }^{94}$. Erhalten sind sowohl ein Mitglieds- wie auch ein Anniversarienverzeichnis des Hospitals von S. Maria in Portico, die allerdings erst nach ca. 1450 einsetzen und bis in die ersten Jahre des 16. Jahrhunderts weitergeführt wurden" ${ }^{95}$. Die nicht mehr - wie in den anderen Beispielen - in Latein, sondern in Volgare geschriebenen Namen spiegeln ein ganz anderes Umfeld wider, als wir es im Falle der beiden vorausgegangenen Hospitalsgründungen kennengelernt haben. Die Personen, die um dieses Hospital 'gravitiertenı, waren Exponenten des gehobenen römischen Bürgertums mit einigen Familien der kommunalen Oberschicht ${ }^{96}$ (wobei der

${ }^{94}$ Pietro Pericol, L'Ospedale di S. Maria della Consolazione di Roma dalle sue origini ai giomi nostri, Imola 1879; Anna EsPosito, Le confratemite e gli ospedali di S. Maria in Portico, S. Maria delle Grazie e S. Maria della Consolazione a Roma (secc. XV-XVI), in: Ricerche di Storia sociale e religiosa n.s. 17-18 (1979) S. 145-172; DIES., S. Maria in Portico, della Consolazione e delle Grazie, arciconfraternita, in: Storiografia e archivi 1985, S. 349-351.

${ }^{95}$ Leider fehlen genauere Jahresangaben, so daß man sich mit einem Abgleich der Namen mit solchen, die auch in den Anniversarienlisten der Salvatorbruderschaft vorkommen, behelfen muß (Doppelmitgliedschaft war ja grundsätzlich möglich: siehe unten Anm. 102): ASR, Ospedale della Consolazione, culto, 5; ed. ist das - nach DYKMANS, Les obituaires (wie Anm. 23), S. 630 in das Jahr 1479 zu datierende - Anniversarienverzeichnis (cc. 50-80) und das - unter Paul II. (1464-1471) angelegte - Mitgliederverzeichnis (cc. 1-49) in EGIDI, Necrologi (wie Anm. 11), I, S. 543-557 und II, S. 533-548.

${ }^{96}$ Man gedenkt besonders des Raffaele Pellegrini, der im Amt eines Konservators der Camera Urbis verstorben war, d. h. als Inhaber eines der höchsten Ämter der römischen Kommune. Als verdienter Guardian der Bruderschaft wurde er von seinen Mitbrüdern feierlich zu Grabe getragen: ibid., II, S. 548. 
Baronaladel ${ }^{97}$ aber völlig fehlte). Nicht minder stark war der Anteil der Mittelschicht von kleineren Händlern und Handwerkern sowie - oft von außerhalb Roms stammenden - Pfarrern und Kaplänen an sekundären Kirchen ${ }^{98}$, während hohe Geistliche und Kuriale bis auf wenige Ausnahmen fehiten. Die Verteilung der Mitglieder auf die einzelnen Stadtteile Roms zeigt ein Übergewicht der Rioni in Tibernähe (Parione, Arenula, S. Angelo, Trastevere und Ripa) sowie von Pigna und Campitelli am Fuße des Kapitols. Mit diesen Stadtvierteln ist der Bereich Roms umschrieben, in dem sich die Bevölkerung und somit das Gewerbe und Handwerk konzentrierten ${ }^{99}$. Bemerkenswert ist, daß die Frauen in einer separaten Rubrik, je nach Rione, aufgeführt sind. Obwohl die römischen Namen weit überwiegen, ist es doch unverkennbar, daß viele Mitglieder zugewanderte Handwerker und Kleinhändler waren ${ }^{100}$. Für sie war der Eintritt in eine Bruderschaft auch ein Mittel, sich sozial in das neue Umfeld zu integrieren. Fremde von jenseits der Alpen waren aber die Ausnahme. ${ }^{101}$ Die Tatsache, daß man etliche Namen auch in den Listen der Salvatorebruderschaft wiederfinden kann, erinnert daran, daß doppelte und mehr Mitglied-

${ }^{97}$ Amtsträger in den Haushalten der Barone werden als solche hervorgehoben, so der Kaplan des Kardinals Orsini, Magister Leonardo aus Velletri, oder der Kämmerer eines Kardinals Savelli und der Kanzler des Mariano Savelli (dieser Baron selbst war dagegen 1468 nicht hier, sondern in die Salvatorbruderschaft aufgenommen worden: ibid., II, S. 468): ibid. S. 536, 547.

${ }^{98}$ "Li nomi delli preti« sind den Laien vorangestellt: EGIDI, Necrologi (wie Anm. 11), II, 535f. Kein einziger Kanoniker der drei großen Basiliken S. Pietro, S. Giovanni in Laterano und S. Maria Maggiore ist verzeichnet. Zur sozialen Herkunft des niederen Klerus sowie des Pfarrklerus in Rom fehlen genauere Untersuchungen, doch deutet vieles darauf hin, daß eine beträchtliche Zahl von ihnen von außerhalb Roms stammte, zumal da die vornehmen Römer die Kanonikate an den bedeutenderen Kirchen der Stadt bevorzugten: REHBERG, Kanoniker (wie Anm. 31), u. a. 138f. Diese Einschätzung wird auch von den nomina sacerdotum et clericorum der Salvator-Bruderschaft bestätigt, unter denen sich viele Nicht-Römer finden: EGIDI, (wie Anm. 11), II, S. 462-467.

${ }^{99}$ Vgl. zur Topographie Roms unter diesem Aspekt als Quelle Egmont LEE. Descriptio Urbis. The roman census of 1527, Rom 1985 (Biblioteca del Cinquecento, 32) und des weiteren u. a. LORI SANFILIPPO, La Roma dei romani (wie Anm. 7); Egmont LEE, Workman and Work in Quattrocento Rome, in: Paul A. RAMSEY (Hg.), Rome in the Renaissance. The City and the Myth. Papers of the Thirteenth Annual Conference of the Center for Medieval \& Early Renaissance Studies, Binghampton/NY 1982, S. 141-152; Ivana Arr, Mercato del lavoro e »forenses« a Roma nel XV secolo, in: Eugenio SONNINO (Hg.), Popolazione e società a Roma dal medioevo all'età contemporanea, Rom 1998, S. 335-358.

${ }^{100} \mathrm{Vgl}$. hier nur den piellimantielli ([Alt-]Kleiderhändler) Giovanni aus Cori, den cartaro Lorenzo aus Ferrara, den miniatore Bartolomeo aus Lucca, ferraro Ambrosio aus Mailand: EGIDI, Necrologi (wie Anm. 11), I, 539, 540, 542. Im Rione Ponte fallen mehrere Venezianer auf: ibid. S. 538. Es kämen noch viele Auswärtige hinzu, wenn man von der berechtigten Annahme ausgeht, daß bei vielen Namen die Herkunft nicht angegeben wurde. Zum Zuzug von Fremden nach Rom siehe das nächste Kapitel.

${ }^{101}$ So fallt ein Martino de Martino, todescho auf: EGIDI, Necrologi (wie Anm. 11), II, 539. 
schaften nicht ausgeschlossen waren, obgleich sie immer mehr verpönt waren $^{102}$. Die meisten Kirchen, an denen Jahrtagsämter gehalten wurden (und in deren Nähe auch die meisten Bruderschaftsmitglieder wohnhaft waren), sind im Tiberknie und in Trastevere zu verorten, was angesichts der Herkunft der meisten Mitglieder nicht überrascht. Eine Ausnahme bildet S. Maria in Aracoeli, die Kirche des traditionell mit der römischen Kommune eng verbundenen Franziskanerkonventes auf dem Kapitol ${ }^{103}$. Hier ließen sich auch viele Römer bestatten, die nicht in der Nähe ansässig waren. Wenig gefragt waren Kirchen der abgelegeneren Stadtteile, wie z.B. des Rione Monti mit seinen beiden nicht berührten Basiliken S. Giovanni in Laterano und S. Maria Maggiore.

Es war aber nicht nur die Sorge um das Seelenheil, die den Eintritt in die Bruderschaft veranlaßte, sondern auch die Möglichkeit, sich in der Kirche eines Hospitals einen herausgehobenen Begräbnisplatz zu sichern. Davon zeugen einige testamentarische Bestimmungen, die Anna Esposito gesammelt hat ${ }^{104}$. Selbst der - relativ - kleine Mann hatte ein ausgeprägtes Bedürfnis, sich der Gebete und memoria der Nachwelt, und sei es nur im Kreise seiner Mitbrüder, zu vergewissern. Auch hier war wichtig, daß man demselben sozialen Milieu angehörte ${ }^{105}$.

\section{Das Hospital der Anima}

Spital einer landsmannschaftlichen Bruderschaft

In Rom, einem Zentrum des internationalen Pilgerwesens und Sitz des Hauptes der Christenheit, konnte es nicht ausbleiben, daß sich Menschen, die eine

${ }^{102} \mathrm{Vgl}$. zu dieser Tendenz ESPOSITO, Men and Women in Roman Confratemities in the Fifteenth and Sixteenth Centuries. Roles Functions, Expectations, in: Nicholas TERPESTRA (Hg.), The Politics of Ritual Kinship. Confraternities and Social Order in Early Modern Italy, Cambridge 2000 (Cambridge Studies in Italian History and Culture [o. Bd.]), S. 82-97, hier S. 84, 87. Stefano de Satro (vgl. Anm. 104) ließ ein Jahrgedächtnis auch von der Salvatorbruderschaft ausrichten: EGIDI, Necrologi (wie Anm. 11), I, S. 442.

${ }^{103}$ Zur Verbindung des Konventes mit dem römischen Stadtregiment vgl. u.a. Giulia BARONE, I Francescani a Roma, in: Storia della città 3/9 (1978) S. 33-35.

104 ESPOSITO, Le confratemite romane (wie Anm. 3), S. 111 f. (insbesondere die beiden Testamente aus dem Jahr 1482) des Stefano de Satro und des sutor (Schuster) Egidio Ippoliti.

${ }^{105} \mathrm{Vgl}$. zur Bedeutung der Schichtzugehörigkeit bei den karitativen testamentarischen Verfügungen u.a. Gunnar MEYER, Milieu und Memoria - Schichtspezifisches Stiftungsverhalten in Lübecker Testamenten aus dem 2. Viertel des 15. Jahrhunderts, in: Zeitschrift des Vereins für Lübeckische Geschichte und Altertumskunde 78 (1998) S. 115-141. So erhielt das Leprosenspital St. Jürgen in Lübeck überdurchschnittliche Zuwendungen von ärmeren Erblassern: ibid. S. 138f., 141. 
gemeinsame Sprache besaßen, solidarisch zusammentaten, wenn sie in dieser Stadt Hilfe oder nur eine vorübergehende Bleibe suchten. Die Pilgerquartiere (scholae peregrinorum) einiger großer nordalpinen Volksstämme (Langobarden, Franken, Friesen und Angelsachsen) sind deshalb schon im Frühmittelalter entstanden, gerieten dann aber in Schwierigkeiten ${ }^{106}$. Das erste Hospital der 'Deutschen', oder besser der Franken, wird mit Karl dem Großen in Verbindung gebracht, ging aber bald ein, sieht man von seinem Begräbnisplatz an der Peterskirche $a b^{107}$. Der Zustrom der Pilger erlebte mit der Einführung der Jubeljahre durch Bonifaz VIII. im Jahre 1300 einen erneuten Aufschwung, der logistische Probleme für die überforderte Stadt Rom mit sich brachte ${ }^{108}$.

Wenn wir uns im folgenden einem prominenten Beispiel eines von NichtRömern getragenen Spitales - nämlich dem von 'Deutschen` begründeten Hospiz der Anima - zuwenden, so geschieht dies nicht mehr mit der nationalgeschichtlichen Perspektive, die lange das Bild dieser Stiftungen in der Literatur geprägt hat ${ }^{109}$. Das besagte Hospiz war aber nicht die erste deutsche karitative Initiative in Rom: der aus dem preußischen Kulm stammende Kleriker

${ }^{106}$ Die Literatur zu den scholae findet sich zitiert in BIRCH, Pilgrimage to Rome (wie Anm. 7), S. 131-133, 140f.; Letizia PANI ERMINI, Dai complessi martiriali alle »civitates«. Formazione e sviluppo dello "spazio cristiano«, in: DIES., Paolo SINISCALCO (Hg.), La comunità cristiana di Roma. La sua vita e la sua cultura dalle origini all'alto medioevo, Città del Vaticano 2000 (Atti e documenti, 9), S. 397-419, bes. 403f.; Letizia PANI ERMINI, La »Schola Saxonum« e le »Scholae Peregrinorum« nella "Civitas Leoniana«, in: L'Antico Ospedale di Santo Spirito 2001, S. 37-46. Zur Entwicklung der Hospitäler mit Herbergsfunktionen vgl. allgemein einige Beiträge in: Hans Conrad PEYER, Von der Gastfreundschaft zum Gasthaus. Studien zur Gastlichkeit im Mittelalter, Hannover 1987 (Monumenta Germaniae Historica. Schriften, 31).

${ }^{107}$ Zur schola Francorum, die an der Salvatorkirche (spärliche Reste von ihr sieht man noch neben dem Palast des Hl. Officiums) zu verorten ist, vgl. Rudolf SCHIEFFER, Karl der Große, die schola Francorum und die Kirchen der Fremden in Rom, in: Römische Quartalschrift 93 (1998) S. 20-37.

${ }^{108}$ Die Literatur zu den Heiligen Jahren in Rom ist auch mit Blick auf das letzte Jubeljahr (2000) noch einmal angeschwollen. Vgl. allgemein Marcello FAGIOLO, Maria Luisa MADONNA (Hg.), La storia dei giubilei, I: 1300-1423; II: 1450-1575, Rom 1997. Zum Pilgerwesen allgemein siehe unten Anm. 177.

${ }^{109}$ Nationales Pathos durchzieht vor allem Joseph SCHMIDLIN, Geschichte der deutschen Nationalkirche in Rom S. Maria dell'Anima, Freiburg i. Br., Wien 1906. Vgl. zu dem genannten Perspektivenwechsel Paul BERBÉE, Von deutscher Nationalgeschichte zu römischer Lokalgeschichte. Der Topos vom »nationalen Pilgerheim « am Beispiel des deutschen Frauenhospizes St. Andreas in Rom (1372-1431), in: Römische Quartalschrift 86 (1991) S. 23-52. Für die Bedeutung der Gemeinsamkeiten von Sprache, Herkunft und Geschichte unter den Mitgliedern der deutschrömischen Bruderschaften, die aber auch interne regional geprägte Gruppenbildungen kannten, vgl. jetzt Knut SCHULZ, Was ist deutsch? Zum Selbstverständnis deutscher Bruderschaften im Rom der Renaissance, in: Andreas MEYER, Constanze RENDTEL, Maria WITTMER-ButSCH (Hg.), Päpste, Pilger, Pönitentiarie. Festschrift für Ludwig Schmugge zum 65. Geburtstag, Tübingen 2004, S. 135-179. 
Nicolaus Henrici ${ }^{110}$, Kaplan an S. Lorenzo in Panisperna, fügte nämlich ab $1372^{111}$ mehrere Häuser in der Nähe von S. Biagio de Oliva (heute Via di Monte della Farina) zu einem Wohnort für ca. 30 fromme Witwen und Jungfrauen zusammen, die - wie ihr geistlicher Gönner selbst - als Pilger von jenseits der Alpen nach Rom gekommen waren und hier ein neues Zuhause und eine neue Lebensaufgabe gefunden hatten. Die traditionelle Annahme, daß der Geistliche sich mit seiner Gründung der deutschen Pilger erbarmt habe, die im Jahre 1350 zum zweiten Jubiläumsablaß oder infolge der Rückkehr der Kurie nach Rom im Jahre 1377 an den Tiber gekommen waren, ist mittlerweile widerlegt ${ }^{112}$. Die anfangs noch relativ geringe Rolle nnationaler Motive wird auch dadurch deutlich, da $\beta$ der Deutsche die Hilfe des Engländers Andreas Alanus für die Errichtung der Hospizkapelle gewann, die seinem Namenspatron geweiht wurde ${ }^{113}$. Obwohl das Andreas-Hospiz als Beginenkonvent einzustufen ist, diente es im Jubeljahr 1390 vorübergehend auch deutschen Pilgern als Anlaufstelle ${ }^{114}$. Nicolaus Henrici blieb seiner Gründung, die er selbst leitete, bis zu seinem Tod im Jahre 1412 verbunden. Danach folgte eine Phase der Mißwirtschaft (so jedenfalls wurde von Seiten wohl auch frauenfeindlicher Kreise kolportiert), die zum Übergang in den Besitz der Anima im Jahre 1431 führte $^{115}$. Festzuhalten ist, daß Nicolaus Henrici und die Frauen von St. Andreas auch eine bislang kaum beachtete Dimension der Zuwanderung nach Rom aus religiös-spirituellen Motiven verkörpern ${ }^{116}$, die von anderer Art war als die eines klassischen Rom-Pilgers, eines Handwerkers oder Kaufmanns.

Die anderen snationalen Spitals-Gründungen, die ab der zweiten Hälfte des 14. Jahrhunderts entstanden, hatten ebenfalls diesem Trend zu entsprechen und außerdem der nicht minder wichtigen Präsenz von Handwerkern und Kurialen Rechnung zu tragen. In der kleinen englischen Kolonie von einigen Dutzenden von Haushalten - zusammengesetzt aus kleinen Straßenhändlern, Rosenkranzverkäufern, Mühlenarbeitern, Schneidern (sutores) und (allerdings wenigen) Kaufleuten - regte sich im Jahre 1362, als die Kurie vorübergehend

${ }^{110}$ BERBÉE, Von deutscher Nationalgeschichte (Anm. 109), bes. 34ff. (zur Person und zu den Anfängen des heute nicht mehr bestehenden Hospizes St. Andreas an der heutigen Via di Monte della Farina).

i" Franz NAGL, Urkundliches zur Geschichte der Anima in Rom. I. Theil der Festgabe zu deren 500-jährigen Bestehen, in: Mittheilungen aus dem Archiv des deutschen Nationalhospizes S. Maria dell'Anima, Rom 1899 (Römische Quartalschrift. Supplementheft, 12), S. 1-88. hier S. 44ff.

${ }^{112}$ BERBÉE, Von deutscher Nationalgeschichte (wie Anm. 109), S. 35.

${ }^{113}$ Ibid. S. $36 f$.

${ }^{114}$ Ibid. S. $38 f$.

115 Vgl. Elisja SchUlte VAN KesSEL, The quietus to a German hospice in Rome. The annexation of Santi Andrea e Birgitta to the Anima (1431), in: Mededelingen van het Nederlands Instituut te Rome 53 (1994) S. 1-17.

${ }^{116}$ Vgl. BERBÉE, Von deutscher Nationalgeschichte (Anm. 109), S. 46f., $51 \mathrm{f}$. 
in Rom weilte, das Bedürfnis, in der Nähe der heutigen Piazza Farnese zu Ehren des hl. Thomas ein Hospiz zu gründen ${ }^{117}$. Natürlich ist zwischen den Gründungen der Katalanen und Kastiliern zu unterscheiden, die auch nach der Union der Königreiche Aragon und Kastilien im Jahre 1479 getrennte Institutionen blieben. Für die Pilger und Armen aus Aragon stifteten zwei aus Barcellona und Mallorca stammende Frauen zwischen 1352 und 1363 zwei Häuser, denen sich 1380 der König von Aragon annahm. Die Kastilier erhalten mit S. Giacomo degli Spagnoli erst Mitte des 15. Jahrhunderts einen festen religiösen Bezugspunkt ${ }^{118}$. Die erst wieder nach 1417 in Rom stärker präsenten Franzosen hatten mehrere Anlaufstellen. 1473 fanden sich vier Einzelbruderschaften (Franzosen, Burgunder, Lothringer und Savoyarden) - in deren Reihen die Handwerker überwogen - in der Bruderschaft "der vier Nationen" beziehungsweise der "Transalpinen« zusammen, während die vornehmeren Kurialen und die Händler curiam Romanam sequentes nur fünf Jahre später die bekanntere Bruderschaft des hl. Ludwig gründeten, die an der eigens errichteten gleichnamigen Kirche ein Spital unterhielt ${ }^{119}$. Selbst die Portugiesen wollten da nicht zurückstehen ${ }^{120}$.

In diesen Kontext ist nun auch das >deutsche< Hospital S. Maria dell'Anima zu verorten. Wie bei den anderen Hospizen steht an seinem Anfang keine von oben angeordnete Staatsaktion (obwohl Kaiser Sigismund $1433^{121}$ und Friedrich III. 1452 auf ihren Romzügen dazu Gelegenheit gehabt hätten); die Initiative ging svon unten aus. Das Hospital wurde um 1398 (dem Jahr eines ersten

${ }^{117}$ Margaret HARVEY, The English in Rome 1362-1420. Portrait of an Expatriate Community, Cambridge 1999 (Cambridge Studies in Medieval Life and Thought. Fourth Series, 45).

118 Justo FERNÁNDEZ ALONSO, Las iglesias nacionales de Espana en Roma. Sus orígenes, in: Anthologica annua 4 (1956) S. 9-96; Manuel VAQUERO PIÑEIRO, Proiezione sociale e risorse economiche di San Giacomo degli Spagnoli alla fine del '400, in: Studi Romani 38 (1990) S. 69-82; DERS., Una realtà nazionale composita: Communità e chiese »spagnole« a Roma, in: Sergio GENSINI (Hg.), Roma capitale (1447-1527), Pisa 1994 (Collana di Studi e Ricerche, $5=$ Pubblicazioni degli Archivi di Stato. Saggi, 29), S. 473-491.

${ }^{119}$ Vgl. Pierre LA CroIX, Mémoire historique sur les institutions de la France à Rome, puisé dans leur archives et autres documents la plupart inédits, 2 éd. révue, annotée et considérablement augmentée par Jean ARNAUD, Rome 1892, S. 41 ff.; Jean-François ARRIGHI, Des confréries françaises aux Pieux Établissements, in: Les fondations nationales dans la Rome pontificale, Rome 1981 (Collection de l'École française de Rome, 52), S. 1-10.

${ }^{120}$ Die erste Gründung eines portugiesischen Hospizes (1363) geht auf eine Frau, Guiomar, zurück, über die nähere Angaben fehlen: Maria DE LURDES PEREIRA ROSA, L'ospedale della nazione portoghese di Roma, sec. XIV-XX. Elementi di storia istituzionale e archivistica, in: Mélanges de l'École française de Rome. Italie et Méditerranée 106 (1994) S. 73-128, hier S. $75 \mathrm{ff}$.

${ }^{121}$ Der Kaiser selbst zeigte sich allerdings nicht sehr großzügig: Clifford W. MAAS ( $\dagger$ ), The German Community in Renaissance Rome, 1378-1523, hg. von Peter HERDE, Freiburg i. B. 1981 (Römische Quartalschrift für christliche Altertumskunde, Supplementheft, 39), S. 83. 
päpstlichen Ablaßbriefes) wohl mit Blick auf das Jubiläumsjahr $1400^{122}$ von einem Ehepaar aus Dordrecht - der Mann, Johann Petri, war ein päpstlicher serviens armorum, also Leibgardist, gewesen - gegründet. Der reiche Kuriale Dietrich von Niem unterstützte die Gründung großzügig. Auf seine Initiative geht wohl auch die Etablierung einer Bruderschaft von in Rom residierenden deutschen Handwerkern und Kurialen an der Anima zurück. Die Mitgliedschaft war formell an keinen Stand oder eine bestimmte Nationalität geknüpft, wobei allerdings die Herkunft aus dem deutschsprachigen Raum (von Ostpreußen bis Brabant und Flandern) dominierte. Viermal im Jahr war eine Spende fällig. Der glücklicherweise erhalten gebliebene Liber confraternitatis der Anima-Bruderschaft, dessen erster Teil erst 1449 bzw. 1463/4 aufgnund von verlorengegangenen Vorlagen von Heinrich Marwede überarbeitet wurde, gibt wieder Aufschluß über ihr Innenleben ${ }^{123}$. Der Liber hat acht, nicht immer scharf getrennte Teile:

Der erste kurze Abschnitt führt die Päpste von Klemens VI., unter dem man die - fiktive - Gründung des Hospitals im Jahre 1350 ansetzte, bis zu Alexander VI. (1342 bis 1503) auf, wobei allerdings nur wenige direkte Bezüge zur Anima (z.B. eine Privilegierung des Hospitals) erwähnt werden ${ }^{124}$. Dieselbe Funktion, das Hospital in direkten Kontakt zu den Spitzen von Kirche und Reich zu bringen, erfüllt die kurze Liste von Kaisern bzw. römischen Königen von Karl IV. bis Maximilian I. ${ }^{125}$. Die Auswahl der Namen hat auch den Zweck, die Gründung der Anima mit dem Jubiläum von 1350 in Verbindung zu bringen. Der dritte Teil enthält nun erstmals die Namen von Persönlichkeiten mit näheren Verbindungen zur Kirche der Deutschen. Dies waren Kardinäle (allen voran Nicolaus Cusanus ${ }^{126}$ ), Erzbischöfe, Bischöfe, Äbte und führende Kuriale, die manchmal hier nur deshalb aufgeführt wurden, weil sie in der

${ }^{122}$ Zur Debatte um das Jahr der Gründung vgl. ibid. S. 70f. und Joseph LENZENWEGER, Sancta Maria de Anima, Erste und zweite Gründung, Wien 1959, S. 12-18.

${ }^{123}$ EGIDI, Necrologi (wie Anm. 11), II, S. 3-105. Für die Einträge in das Bruderschaftsbuch nach 1500 ist immer noch die Edition von Karl JAENIG, Liber confraternitatis b. Marie de Anima Teutonicorum de Urbe ..., Romae 1875 heranzuziehen. Im Archiv der Anima habe ich eine fotomechanische Reproduktion konsultiert. Zum Aufbau und zur Einfuihrung vgl. Alois LANG, Studien zum Bruderschaftsbuche und den ältesten Rechnungsbüchern der Anima in Rom. II. Theil der Festgabe zu deren 500-jährigen Bestehen, in: Mittheilungen aus dem Archiv 1899, S. 89-155 (S. 96 zum Anteil Marwedes); MAAS, The German Community (wie Anm. 121), S. 148ff; Christiane SCHUCHARD, Die Deutschen an der päpstlichen Kurie im späten Mittelalter (1378-1447), Tübingen 1987 (Bibliothek des Deutschen Historischen Instituts in Rom, 65), S. 326-329 (mit Korrekturen an den Ergebnissen von Maas).

${ }^{124}$ EGIDI, Necrologi (wie Anm. 11), II, S. 9-11.

${ }^{125}$ Ibid. S. 11 f.

${ }^{126} \mathrm{Vgl}$. zu seiner großzügigen Stiftung zugunsten der Anima Hermann J. HaLLAUER, Das St. Andreas-Hospiz der Anima in Rom. Ein Beitrag zur Biographie des Nikolaus von Kues, in: Mitteilungen und Forschungsbeiträge der Cusanus-Gesellschaft 19 (1991) S. 25-52. 
1431 errichteten Kirche (die noch einmal ab 1499 durch einen prächtigen Neubau ersetzt wurde ${ }^{127}$ ) ihre Bischofsweihe erhalten hatten ${ }^{128}$. Der vierte Teil ist Königen, Herzögen, Markgrafen, Grafen, Baronen und anderen Adeligen vorbehalten ${ }^{129}$. Man erkennt sofort, daß es sich bei diesen Herren meist um vornehme Pilger (zumal in den Jahren 1450 und 1500) oder Begleiter der beiden vorgenannten Kaiser handelt. Die eigentlichen in Rom wohnhaften Vollmitglieder der Bruderschaft findet man eher in den folgenden Rubriken: In der fünften sind die Kleriker und Kuriale verzeichnet ${ }^{130}$. Die sechste Sektion ist den verstorbenen Geistlichen reserviert ${ }^{131}$. Die nächsten beiden Teile (der siebte und achte ${ }^{132}$ ) umfassen die lebenden und verstorbenen Laien - darunter viele Frauen! - unter den Mitbrüdern und Wohltätern. Nach $1475^{133}$, als der Anteil der Laien zurückging, wurden hier auch verstorbene Geistliche eingetragen.

Da die Verzeichnisse nicht immer chronologisch geordnet sind und einige Mehrfachnennungen und sonstige Widersinnigkeiten enthalten ${ }^{134}$, ist ihre statistische Auswertung sehr schwierig. Um letzte Klarheit zu gewinnen, bedarf es einer Neuedition des Liber fraternitatis; Christiane Schuchard hat seine Einträge mit dem noch unedierten Liber receptorum abgeglichen. In diesem ältesten Einnahmebuch mit Einträgen von 1426 bis $1515^{135}$ wurden die Teilnehmer an den Mitgliedsversammlungen der Anima-Bruderschaft und ihr finanzieller Beitrag verzeichnet. In dem oben genannten Intervall (genauer von 1428 bis 1514) ist der Beitritt von insgesamt 90 männlichen Laien, von 45 Frauen und dreimal so vielen Klerikern (433) dokumentiert. Die zeitliche

${ }^{127}$ Barbara BAuMÜLleR, S. Maria dell'Anima Santa Maria dell'Anima in Rom. Ein Kirchenbau im politischen Spannungsfeld der Zeit um 1500. Aspekte einer historischen Architekturbefragung, Berlin 2000.

${ }^{128}$ EgIDI, Necrologi (wie Anm. 11), II, S. 12-23.

${ }^{129}$ Ibid. S. 23-28. Auffallend ist, daß der Liber fraternitatis die Amtsinhaber deutscher Städte mit römischen Amtstiteln wie senatores, conservatores und proconsules versieht. Immerhin findet sich ein baro romanus (Guglielmo Savelli): ibid. S. 23.

${ }^{130}$ Ibid. S. 50-69. Da MAAS, The German Community (wie Anm. 121), S. 149 aus nicht ersichtlichem Grund von einer Trennung unserer fünften Rubrik in eine 5. und 6. "ssection" ausgeht, kommt er auf eine Gesamtzahl von neun Rubriken.

${ }^{131}$ EGIDI, Necrologi (wie Anm. 11), II, S. 69-91.

${ }^{132}$ Ibid. S. 92-96 und 96-105.

${ }^{133} \mathrm{Vgl}$. ibid. S. 103-105 (die ersten hier verzeichneten Geistlichen waren 1478 gestorben).

${ }^{134}$ MAAS, The German Community (wie Anm. 121), S. 131-173.

${ }^{135}$ Christiane SCHUCHARD, Die deutschen Kurialen und die Anima-Bruderschaft in der zweiten Hälfte des 15. Jahrhunderts, in: Stephan Füssel (Hg.), Deutsche Handwerker, Künstler und Gelehrte im Rom der Renaissance. Akten des interdisziplinären Symposions vom 27. und 28.5.1999 im DHIR, Pirckheimer Jahrbuch fur Renaissance- und Humanismusforschung 15/16, Wiesbaden 2001, S. 26-45, hier S. 4. Frau Schuchard hat die Eintritte in die Bruderschaft von 1428 bis 1460 bereits in DIES., Die Deutschen (wie Anm. 123), S. 329-346 analysiert. 
Verteilung verfeinert nur das bereits Gesagte: besonders auffallend ist der $\mathrm{Zu}$ wachs der Geistlichen nach 1450, der die Dominanz der Laien in den Jahren 1434 bis 1443 ablöste $^{136}$. Der Vergleich zwischen den Eintragungen des Liber receptorum mit denen des Liber confraternitatis zeigt, daß letzterer 29 datierte Einträge hat, die im Liber receptorum fehlen. Es handelt sich um Ehefrauen und Kinder, die von einem Haushaltsvorstand abhingen (der als Beitragszahler figuriert), sowie Personen, die bei ihrer Aufnahme kein Geld, sondern Sachoder Arbeitsleistungen erbrachten ${ }^{137}$. Im Verlauf der zweiten Hälfte des 15. Jahrhunderts geht die Gesamtzahl der Beitragszahler von rund 60 Mitgliedern zurück und erreicht ihren Tiefststand am Anfang des 16. Jahrhunderts mit nur noch vier bis fünf Mitgliedern ${ }^{138}$. Der zeitliche Längsschnitt belegt die größere Seßhaftigkeit der Handwerker, während die Kleriker oft nicht so lange in Rom verblieben ${ }^{139}$. Trotz der eklatanten Dominanz der Kurialen ist aber vor vorschnellen Schlüssen aus diesen Resultaten und der voreiligen Konstruktion eines Gegensatzes Kurialen-Handwerker zu warnen, zumal es zwischen beiden Gruppen eine Reihe von verwandtschaftlichen, sozialen und wirtschaftlichen Kontakten gab $^{140}$.

Obgleich es richtig ist, daB sich nun viele durchreisende Geistliche und Adelige in das Bruderschaftsbuch der Anima eintrugen, ist es unangemessen, dieses Buch als »a guest book ${ }^{141}$ zu bezeichnen. Rein formal gab es keinen Unterschied zwischen aktiven Bruderschaftsmitgliedern in Rom und solchen Mitbrüdern in der Ferne. Klar, daß letztere nicht am geistlichen Gemeinschaftsleben (Gottesdiensten, Begräbnissen) an der Anima teilnehmen konnten; sie sahen sich dennoch - wie im Falle der Hospitalsbruderschaft von S. Spirito in Sassia - als Mitbrüder und erwarteten dieselben spirituellen Gnaden (Ablaß, Gebetsgedenken), die den in Rom Wohnenden zukamen. Wie im Falle des Heiliggeist-Krankenhauses profitierte auch die Anima von den Hei-

${ }^{136}$ Ein Grund für das damalige Übergewicht der Laien war zweifellos die Abwesenheit der Kurie aus Rom, gehörten ihr doch zahlreiche deutsche Kleriker - und damit potentielle Mitglieder der Anima - an: Christiane SCHUCHARD, Die Anima-Bruderschaft und die deutschen Handwerker in Rom im 15. und frühen 16. Jahrhundert, in: Knut SCHULZ unter Mitarbeit v. Elisabeth MÜLLER-LUCKNER (Hg.), Handwerk in Europa vom Spätmittelalter bis zur Frühen Neuzeit, München 1999 (Schriften des Historischen Kollegs. Kolloquien, 41), S.1-25, hier S. 5 (auch zu den genannten Zahlen). Zu einer weiteren Analyse des sozialen Wandels in der Zusammensetzung der Bruderschaft vgl. SCHULZ, Confraternitas Campi Sancti (wie Anm. 5), S. 50-52.

${ }^{137}$ SCHUCHARD, Die Anima-Bruderschaft (wie Anm. 136), S. 8.

${ }^{138}$ Ibid. S. $10 \mathrm{ff}$.

${ }^{139}$ Ibid. S. 13 f.

${ }^{140}$ Ibid. S. $20 \mathrm{ff}$.

${ }^{141}$ MAAS, The German Community (wie Anm. 121), S. 92, 94. Vgl. schon die Klarstellung in SCHUCHARD, Die Deutschen (wie Anm. 123), S. $328 f$. 
ligen Jahren, nur, daß in ihrem Fall es vorwiegend Deutsche waren, die sich in die Bruderschaft einschrieben ${ }^{142}$.

Die Deutschen verfügten aber noch - sieht man von den Bruderschaften ihrer Handwerksgruppen ab - über eine weitere geistliche Sozietät: die Bruderschaft am Campo Santo Teutonico neben der Peterskirche. Sie wurde erst 1454 für Deutschsprachige gegründet und unterhielt anfangs ebenfalls ein Hospital. ${ }^{143}$ Ihre Mitgliedslisten setzen aber erst im Jahre 1500 ein $^{144}$. Sie war aber schon vorher gerade bei den einfachen Leuten und insbesondere den in Rom ansässigen Handwerkern - die man an der Anima verpönte - sehr beliebt, zumal man sich hier auch günstig bestatten lassen konnte ${ }^{145}$. Und es war dieses Anliegen der Einbindung in die Gemeinschaft der Lebenden und Toten «, das einem Grundbedürfnis der Deutschen (und nicht nur dieser!) in der Fremde entsprach und den Erfolg der Bruderschaft am Campo Santo Teutonico ausmachte, wobei auch das Gesellige und das Gemeinschaftsbewußtsein gepflegt wurden ${ }^{146}$. Dagegen stagnierte - wenn auch auf hohem sozialen Niveau - die Anima-Bruderschaft, die über keinen großen Friedhof verfügte. Die Krankenpflege trat dagegen in beiden Bruderschaften (aber auch bei anderen ähnlich strukturierten snationalen، Stiftungen) bald in den Hintergrund, was dazu führte, daß die Hospize der Fraternitäten dieses Typs sich nicht zu eigentlichen Krankenhäusern entwickelten. Der landsmannschaftliche Zusammenschluß, der sich von der Stadt Rom und ihrer Bevölkerung abkehrte, versperrte ihnen die Chance einer wirtschaftlichen Expansion, die erst den Unterhalt einer anspruchsvolleren Krankenpflege gewährleistet hätte. Hinzu kommt, daß sich mitunter gleich mehrere Bruderschaften, die sich an dieselbe landsmannschaftliche Klientel wandten, gegenseitig Konkurrenz machten; es

\footnotetext{
${ }^{142}$ MAAS, The German Community (wie Anm. 121), S. 97 f.

${ }^{143}$ SCHULZ, Confratemitas Campi Sancti (wie Anm. 5), S. 46f., weist aber darauf hin, daß die um 1490 verfaßten »ersten « Statuta et capitula der Bruderschaft keine Aussagen mehr über eine Armen- und Krankenfürsorge enthalten. Das Hospital war aufgegeben worden, da seine Führung vom mächtigen Ospedale di S. Spirito beansprucht wurde. Erst 1511 schritt man zum Bau eines - eigenen - kleinen Hospitals.

$144 \mathrm{Vgl}$. zu den Anfängen der Bruderschaft ibid. sowie MAAS, The German Community (wie Anm. 121), S. 114-125; Albert Weiland, Der Campo Santo Teutonico in Rom und seine Grabdenkmäler, Rom u.a. 1988 (Römische Quartalschrift für christliche Altertumskunde. Supplementheft, 43), S. 57ff., 61 Anm. 116 (zu den Mitgliedslisten); Knut SCHULZ, Die Anfänge der Bruderschaft des Campo Santo Teutonico (bis zum ersten Viertel des 16. Jahrhunderts), in: Römische Quartalschrift 93 (1998) S. 38-61, bes. S. 56-60 (prosopographische Analyse).

${ }_{145}$ Allerdings wurde der Campo Santo als Bestattungsplatz erst attraktiv, als 1500 die neue Kirche der Bruderschaft eingeweiht wurde. Zuvor ließen sich viele deutsche Handwerker an S. Gregorio al Celio bestatten, da das dortige Olivetaner-Kloster großes Ansehen genoß: MAAS, The German Community (wie Anm. 121), S. 88; SCHUlz, Die Anfänge (wie Anm. 144), S. 46, 55f.; SCHUlZ, Confraternitas Campi Sancti (wie Anm. 5), S. $55 f$.

${ }^{146}$ Ibid. S. 56f.; SCHULZ, Die Anfänge (wie Anm. 144), S. 61 (hier Zitat).
} 
gab ja auch noch eigene deutsche Handwerkerbruderschaften wie die der Bäkker, Weber und Schuhmacher etc. ${ }^{147}$. Zu erinnern ist auch an eine bereits vor 1427 existierende Bruderschaft der sprachverwandten Flamen, die ein eigenes Hospiz bei S. Giuliano >dei Fiamminghi unterhielten ${ }^{148}$. Diese KonkurrenzSituation, die natürlich auch gewisse soziale, kulturelle, politische und sprachliche Differenzen widerspiegelt, haben wir ja schon bei den Franzosen und Spaniern angetroffen ${ }^{149}$. Aber auch die Florentiner hatten mehrere Konfraternitäten (Confraternita della Pietà di S. Giovanni dei Fiorentini, di S. Giovanni Decollato usw.) ${ }^{150}$. Die einzelnen landsmannschaftlichen Bruderschaften gingen auch in den nachfolgenden Jahrhunderten oft separate Wege, wobei ihre unterschiedliche Bindung an die Monarchen ihrer Heimatländer hervorgehoben sei: Die Könige der Nationalstaaten Frankreich und Spanien spielten Ende des 15. und Anfang des 16. Jahrhunderts eine größere Rolle als das Reich bzw. der Kaiser für die Anima ${ }^{151}$.

${ }^{147}$ Vgl. Knut ScHulz, Deutsche Handwerkergruppen im Rom der Renaissance. Mitgliederstärke, Organisationsstruktur, Voraussetzungen. Eine Bestandsaufnahme, in: Römische Quartalschrift für christliche Altertumskunde 86 (1991) S. 3-22; SCHUCHARD, Die AnimaBruderschaft (wie Anm. 123), S. 17f. Zu den Deutschen in der internationalen Heiliggeistbruderschaft vgl. Anm. 39.

${ }_{148} \mathrm{Vgl}$. Maurice VAES, Les fondations hospitalières flammandes à Rome du $\mathrm{XV}^{\mathrm{e}}$ au XVIr siècle, Bulletin de l'Institut historique belge de Rome 1 (1919) S. 161-371; Elisja SCHULTE VAN KESSEL, Le istituzioni fiamminghe e olandesi a Roma durante il Rinascimento, in: Anne-Claire DE LIEDEKERKE (Hg.), Fiamminghi a Roma 1508-1608; artisti dei Paesi Bassi e del Principato di Liegi a Roma durante il Rinascimento, Mailand 1995, S. 61-66.

${ }^{149} \mathrm{Vgl}$. insgesamt die instruktive Karte $\mathrm{zu}$ den mationalen « Bruderschaften und Handwerkerbruderschaften in Knut SCHULZ, Deutsche Handwerkergruppen im Rom des 15. und beginnenden 16. Jahrhunderts, in: FüsSEL, Deutsche Handwerker (wie Anm. 135), S. 11-25, hier S. 25.

iso Vgl. Claudia CONFORTI, La »natione fiorentina« a Roma nel Rinascimento, Donatella CALABI, Paola LANARO ( $\mathrm{Hg}$.), La città italiana e i luoghi degli stranieri (XIV-XVIIl secolo), Rom 1998 (Biblioteca di cltura moderna, 1141), S. 171-191; Irene POLVERINI FOSI, I fiorentini a Roma nel Cinquecento: storia di una presenza, in: Roma capitale (wie Anm. 118), S. 389-414. Zur Konkurrenzsituation der landsmannschaftlichen Bruderschaften untereinander vgl. Anna ESPOSITO, I »Forenses« a Roma nell'età del Rinascimento: Aspetti e problemi di una presenza »atipica«, in: Gabriella ROSSETTI (Hg.), Dentro la città. Stranieri e realtà urbane nell'Europa dei secoli XII-XVI, Neapel 1993, S. 163-175, überarbeitet in: DIES., Un'altra Roma Minoranze nazionali e comunità ebraiche tra Medioevo e Rinascimento, Rom 1995, S. 75-92, hier vor allem S. 89. Die Präsenz von Italienern aus anderen Regionen und Städten in Rom kann hier nicht vertieft werden. Stellvertretend für die Vielfalt dieser Gruppen sei auf Giuseppe BONACCORSO, I veneziani a Roma da Paolo II alla caduta della Serenissima: l'ambasciata, le fabbriche, il quartiere, in: La città italiana. S. 192205 hingewiesen.

${ }^{151}$ Als Beispiel sei auf den Einfluß der spanischen Könige auf ihre in Rom weilenden Untertanen hingewiesen: vgl. Alessandro SERIO, Modi, tempi e uomini della presenza hispana a Roma nel primo Cinquecento (1503-1527), in: Francesca CANTU், Maria Antonietta VISCEGLIA (Hg.), L'Italia di Carlo V. Progetti, politiche di governo e resistenze all'impero nell'età 


\section{Schlußbetrachtung}

Mehr als der meist allgemein gehaltene Wortlaut der Statuten ermöglicht im Falle der Stadt Rom die prosopographische Analyse der Mitglieder der sie tragenden Bruderschaften die Rekonstruktion des sozialen Hintergrunds der Entstehung und indirekt auch des eigenen Geschichtsbildes, der Ziele und der Bedürfnisse der unterschiedlichen Stifter-Kreise und ihrer Hospitäler. Erst die Verankerung der karitativen Stiftungen in die gesellschaftlichen und wirtschaftlichen Gegebenheiten der sie umgebenden Stadt, gewährleistete ihnen unabhängig davon, ob sie päpstlicherseits oder von privater Seite initiiert worden waren - einen bleibenden Erfolg. Obgleich im Rahmen der vorliegenden Betrachtung einige nicht minder wichtige Faktoren für die Gründungsgeschichte der besagten Einrichtungen (wie die noch einer modernen Untersuchung harrende Privilegierung durch die Päpste ${ }^{152}$ ) nur am Rande behandelt wurden und trotz der Beschränkung auf nur vier Beispiele ${ }^{153}$, lassen sich aus dem Gesagten dennoch einige allgemeine Feststellungen zu unserer Ausgangsfrage treffen. Als Motive der Hospitalsgründungen in Rom erkennt man - wie anderenorts - das Bedürfnis nach einer sanitären Grundversorgung und den religiös motivierten Drang zum Dienst am Nächsten und zur Sicherung des Seelenheils sowie den Wunsch nach einer sozialen oder landsmannschaftlichen Gemeinschaftsbildung in Form einer Bruderschaft. Hinzu kommen auch Rom-spezifische Bedingungen, wie die dominante Rolle des Papstes als Stadtherr, aber auch Schirmherr der Pilger und Kurialen, die dem schwachen römischen Stadtregiment keinen Entfaltungsspielraum beließ. War das Ordenskrankenhaus von S. Spirito das Hospital der Kurie, so übernahmen die vorrangig von Laien getragenen Häuser der Salvatorbruderschaft - wenn auch erst recht spät - kommunale Versorgungsaufgaben. Beide Institutionen waren zeitweise starken Pressionen des römischen Adels ausgesetzt. Der starke Einfluß des päpstlichen Stadtherrn und des Baronaladels auf Rom bedingten den

di Carlo V. Atti del congresso internazionale (Roma, 3-4-5 aprile 2001), Rom 2003, S. 433476. Immerhin wurde 1518 die Anima von Kaiser Maximilian unter den Schutz des Reiches gestellt. LENZENWEGER, Sancta Maria (wie Anm. 122), S. 27.

${ }^{152}$ Unzulänglich ist Benedetto DA ALATRI, Gli ospedali di Roma e le bolle pontificie (Aspetti giuridici), Viterbo 1950. Für S. Spirito vgl. REHBERG, I papi, l'ospedale (wie Anm. 17), S. 42-53.

${ }^{153}$ So konnte nicht auf die nicht minder wichtigen, wenn auch kleineren Hospitäler wie S. Giacomo in Augusta unweit der Piazza del Popolo (s. Anm. 56), das Magdalenen-Hospital in der Nähe des Pantheon oder die Hospitäler der unter dem Namen Gonfalone vereinten Bruderschaften eingegangen werden: vgl. zu ihnen DA ALATRI, Il medio evo (wie Anm. 7), S. 161-179; Anna ESPOSITO, Le confraternite del Gonfalone (secoli XIV-XV), in: Le confraternite romane 1984, S. 105-136; DIES., Gli ospedali romani (wie Anm. 3), S. 24lf. (mit weiterführender Literatur). 
besonderen Charakter der römischen Hospitallandschaft, die den Trend zur 'Kommunalisierung nicht mitmachte, den man besonders für die deutschen Lande ${ }^{154}$, aber auch für die Stadtrepubliken Florenz, Venedig, Mailand, Bologna $^{155}$ etc. betont hat. Besonders Florenz wird eine Vorreiterrolle zuerkannt, die auf das Fehlen von "competing forces « wie einer starken Monarchie, eines Hofes oder eines ausgeprägten Stadtadels zurückgeführt wird, die die Ausprägung eines kommunalen Gesundheitswesens behindern hätten können. ${ }^{156}$ Es ist für das mangelnde Engagement - oder besser: den mangelnden Spielraum der römischen Kommune bezeichnend, daß das einzige in den Stadtstatuten von 1360/63 namentlich genannte Hospital S. Spirito in Sassia ist; inm wird die Exemtion von Abgaben zugesichert ${ }^{157}$. In Nachahmung des Vorbildes, das Cola di Rienzo gegeben hatte ${ }^{158}$, verfügten die Statuten immerhin, daß sich der Senator der Hospitäler, der Witwen, Minderjährigen und Armen anzunehmen habe ${ }^{159}$. Dieses erste Engagement hängt natürlich mit der Abwesenheit der

${ }^{154}$ Maßgeblich hierfür ist immer noch REICKE, Das deutsche Spital (wie Anm. 6), I, S. 196277 (Reicke spricht vom »Prozeß der Verbürgerlichung des Spitalwesens «), II, S. 53-116 ( Das bürgerliche Spital«). Vgl. Uta LINDGREN, Hospital, in: Lexikon des Mittelalters, V, München, Zürich 1991, Sp. 133-137 und - mit einer differenzierenden Sicht - Gisela DROSSBACH, Das Hospital - eine kirchenrechtliche Institution? (ca. 1150-ca. 1350), in: Zeitschrift der Savigny-Stiftung für Rechtsgeschichte. Kanonistische Abteilung 118 (2001) S. 510-522, hier S. 517f.

${ }^{155} \mathrm{Vgl}$. hier nur - aus einer reichen Literatur - Brian PULLAN, Rich and Poor in Renaissance Venice. The Social Institutions of a Catholic State, to 1620, Oxford 1971, S. 197ff. (ital. Übersetzung: La politica sociale della Repubblica di Venezia, 1500-1620, Rom 1982); Philip GavITT, Economy, Charity, and Community in Florence, 1350-1450, in: Aspects of Poverty in Early Modern Europe, ed. Thomas RIIS, Florenz 1981, S. 79-118; DERS., Charity and Children in Renaissance Florence. The Ospedale degli innocenti, 1410-1536, Ann Arbor 1990 (Studies in Medieval and Early Modern Civilization), S. 13, 61ff.; John HENDERSON, Piety and Charity in Late Medieval Florence, Oxford 1994, S. 241-252, 354-410; Nicholas TERPESTRA, Lay Confratemities and Civic Religion in Renaissance Bologna, Cambridge 1995 (Cambridge Studies in Italian History and Culture [o. Bd.]), S. 179ff.; Giuliana ALBINI, La riforma quattrocentesca degli ospedali nel Ducato di Milano, tra poteri laici ed ecclesiastici, in: Vera ZAMAGNI (Hg.), Povertà e innovazioni istituzionali in Italia. Dal Medioevo ad oggi, Bologna 2000, S. 95-109.

${ }_{156}$ Vgl. BECKER, Aspects of Lay Piety (wie Anm. 19), bes. S. 182f., 198. Ibid. S. 184 unterstreicht nochmals, daß ein Hauptgrund für die idemocratization of spiritual life in der Tatsache $\mathrm{zu}$ suchen sei, sthat the city had no proper nobility to monopolize the spiritualities «.

${ }^{157}$ Vgl. Camillo RE, Statuti della città di Roma, Rom 1883 (Biblioteca dell'Accademia storico-giuridica, 1), S. 156; Alain DE BOŨARD, Le régime politique et les institutions de Rome au Moyen-Ấge, 1252-1347, Paris 1920 (Bibliothèque des Écoles françaises d'Athènes et de Rome, 118), S. 195f.

${ }^{158}$ Die von Cola erlassenen ordinamenti dello buono stato sahen Zahlungen der städtischen Kasse an Witwen und Waisen vor: ANONIMo Romano, Cronica (wie Anm. 54), S. 155.

159 Nach seinem Amtseid gehört es zu seinen Pflichten, whospitalia et religiosa et pia loca manutenere et defensare, et in causis eorum ac viduarum, pupillorum, pauperum et miserabi- 
Kurie in Avignon zusammen. Anders als in den zu diesem Punkt zum Teil sehr ausführlichen Statuten anderer mittel- und norditalienischen Städte ${ }^{160}$, geht der römische Text über die zitierte allgemeine Absichtserklärung nicht hinaus. $\mathrm{Zu}$ gering waren die finanziellen und politischen Mittel der Kommune, um in diesem Bereich gestalterisch tätig zu werden. Allerdings setzen für Rom erst 1515 die Stadtratsprotokolle ein, die jedoch ebenfalls zeigen, daß der Magistrat direkt nur eingriff, wenn eine Pestwelle die öffentliche Ordnung gefährdete ${ }^{161}$. Wie um sich bewußt von der päpstlicherseits favorisierten Organisationsstruktur des Hospitals von S. Spirito in Sassia abzusetzen, folgte man immerhin in der Mitte des 15. Jahrhunderts am Erlöser-Krankenhaus dem Vorbild des Florentiner Hospitals S. Maria Nuova ${ }^{162}$. Noch in der frühen Neuzeit war das Sanitärwesen in Rom fest in der Hand von Bruderschaften (von denen es Ende des 16. Jahrhunderts 107 gab, die sich immer spezielleren Aufgaben zuwandten), während der päpstliche Stadtherr - insbesondere über die Gewährung von Privilegien - nur mäßig dirigierend eingriff ${ }^{13}$.

Relativ frei von kurialer und kommunaler Bevormundung entwickelte sich dagegen eine Vielzahl von kleineren Hospizen, für die das Spital der Consolazione und das der Anima repräsentativ sind. Letztere war eine snationale Gründung. Daß man sich das Europa im Mittelalter im wahrsten Sinne des Wortes sin Bewegung، vorstellen muß, daß es geprägt war von einem steten Kommen und Gehen von Kaufleuten, Geistlichen, Söldnern, Pilgern und

lium personarum et dotum mulierum procedere et procedi facere «: RE, Statuti (wie Anm. 157), S. 205f. (III § 9), vgl. S. 79 (I § CXXII).

${ }^{160}$ In diesem Zusammenhang sei nur auf das Mailänder und Florentiner Gegenbeispiel - mit ihren reichen Quellen - verwiesen: Angelo Francesco LA CAVA, Igiene e sanità negli statuti di Milano del sec. XIV, Mailand 1946 (Studi di storia della medicina, 3); BECKER, Aspects of Lay Piety (wie Anm. 19), S. 177ff.; HeNDERson, Piety and Charity (wie Anm. 155), S. 241-410. Vgl. allgemein auch André VAUCHEZ, Assistance et charité en Occident, XIII ${ }^{\mathrm{C}}$ $\mathrm{XV}^{\mathrm{e}}$ siècle, in: Vera BARBAGLI BAGNOLI ( $\mathrm{Hg}$.), Domanda e consumi. Livelli e strutture (nei secoli XIII-XVIII), Florenz 1978, S. 151-162; ALBINI, Città e ospedali nella Lombardia (wie Anm. 19); Ospedali e città 1997 (darin u. a. Charles M. DE LA. RONCIÈRE, Città e ospedali: bilancio di un convegno, S. 255-272). Vgl. außerdem Gian Maria VARANiNi, Per la storia delle istituzioni ospedaliere nella città della Terraferma veneta nel Quattrocento, in: ibid. S. 107-178, hier S. 120f., $131 \mathrm{f}$.

${ }_{161} \mathrm{Vgl}$. Andreas REHBERG, Die ältesten erhaltenen Stadtratsprotokolle Roms (1515-1526). 3 Teile, in: Quellen und Forschungen aus italienischen Archiven und Bibliotheken 80 (2000) S. 266-359; 81 (2001) S. 278-350; 82 (2002) S. 231-403, hier II, Nr. 152a und III ad indicem (Pest).

${ }^{162}$ EsPOSITO, Gli ospedali romani (wie Anm. 3), S. 240.

${ }^{163}$ Vgl. Paolo SiMONCELLI, Note sul sistema assistenziale a Roma nel XVI secolo. in: Timore e carita (Anm. 4), S. 137-156, bes. S. 143 (zur Zahl der Bruderschaften); Christopher F. BLACK, Italian Confraternities in the Sixteenth Century, Cambridge u.a. 1989, S. 190-196 (ital. Übersetzung: Le confraternite italiane del Cinquecento, Mailand 1992). 
Handwerkern, ist mittlerweile eine allgemein akzeptierte Tatsache ${ }^{164}$. Bekannt sind die italienischen und hansischen Kaufmannskolonien ${ }^{165}$. In vielen Städten Europas gab es Handelskontore und von auswärtigen Handwerkern und Spezialisten $^{166}$ geprägte Straßenzüge ${ }^{167}$. Aber so unbestritten es ist, daß sich diese Gruppen oft bruderschaftlich organisierten und bedürftige Landsleute zu unterstützen bereit waren, so muß man doch feststellen, daß die (medizinische) Grundversorgung und Beherbergung der unbemittelten Fremden in Rom eine besondere Herausforderung waren. Diesen Aufgaben stellte sich dagegen beispielsweise die von deutschen Handwerkern getragene S. Barbara-Bruderschaft in Florenz nicht; anders verhielt es sich wiederum in Trient, das als wichtige Zwischenstation für den Handel über die Alpen und aufgrund der Nähe zum deutschen Sprachraum einen hohen deutschen Einwohneranteil besaB und viele Durchgangsreisende zu bewältigen hatte. Hier wurden Pilger und Wandergesellen auch von örtlichen deutschen Bruderschaften, zumal im Ospedale Alemanno, mitversorgt. ${ }^{168}$ Die Gepflogenheiten hansischer und italienischer

${ }^{164}$ Aus einer breiten Literatur sei nur verwiesen auf die Sammelbände Unterwegssein im Spätmittelalter, hg. von Peter MORAW, Berlin 1985 (Zeitschrift für historische Forschung. Beiheft, 1); Simonetta CAVACIOCCHI (Hg.), Le migrazioni in Europa, sec. XIII-XVIII. Att1 della »Venticinquesima Settimana di Studi«, 3-8 maggio 1993, Florenz 1994 (Istituto internazionale di storia economica $» F$. Datini«. Serie II, Atti delle $"$ Settimane di studio« e altri Convegni 25) und Siegfried DE RACHEWILTZ, Josef RIEDMANN (Hg.), Kommunikation und Mobilität im Mittelalter. Begegnungen zwischen dem Süden und der Mitte Europas (11.14. Jahrhundert), Sigmaringen 1995. Für Italien nun grundlegend: Uwe ISRAEL, Fremde aus dem Norden. Transalpine Zuwanderer im spätmittelalterlichen Italien, Tübingen 2005 (Bibliothek des Deutschen Historischen Instituts in Rom, 111).

${ }^{165} \mathrm{Vgl}$. Amold ESCH, Viele Loyalitäten, eine Identiät. Italienische Kaufmannskolonien im spätmittelalterlichen Europa, in: Historische Zeitschrift 254 (1992) S. 581-608 sowie die Sammelbände Dentro la città (wie Anm. 150) und La città italiana (wie Anm. 150).

${ }^{166}$ Die Deutschen waren beispielsweise vor allem als Bäcker, Weber, Herbergswirte und Schuhmacher renommiert. Die klassische Studie für die Präsenz der Deutschen in Italien ist immer noch Alfred DOREN, Deutsche Handwerker und Handwerkerbruderschaften im mittelalterlichen Italien, Berlin 1903. Vgl. jetzt - wenn auch stark auf Rom bezogen - Knut SCHULZ, Deutsche Handwerker in Italien, in: Kommunikation und Mobilität 1995, S. 115-133. ${ }^{167}$ Vgl. als Beispiel Philip BRAUNSTEIN, Appunti per la storia di una minoranza: la popolazione tedesca di Venezia nel Medioevo, in: Rinaldo COMBA, Gabriella PICCINNI, Giuliano PINTO (Hg.), Strutture familiari, epidemie, migrazioni nell'Italia medievale. Atti del convegno internazionale problemi di storia demografica nell'Italia meridionale (Siena, 28-30 gennaio 1983), Neapel 1984 (Nuove ricerche di storia, 2), S. 511-517. Das Phänomen der Konzentration der deutschen Handwerker auf ganz bestimmte Berufsfelder hängt wohl auch mit dem um 1400 aufkommenden Gesellenwandern zusammen: SCHULZ, Deutsche Handwerkergruppen (wie Anm. 147), S. 21 f.

${ }^{168}$ Die Weber aus den deutsch-flämischen Landen organisierten sich in Florenz erstmals in der Mitte des 14. Jahrhunderts in einer Bruderschaft, die 1446 vom Florentiner Stadtregiment offiziell anerkannt wurde: Mario BATTISTINI, La Confrérie de Sainte-Barbe des Flamands à Florence. Documents relatifs aux tisserands et aux tapissiers, Bruxelles 1931, S. 6ff.; Franco FRANCESCHI, I tedeschi e l'Arte della lana a Firenze fra Tre e Quattrocento, 
Kaufleute in Brügge und Antwerpen ${ }^{169}$ (aber man könnte auch an die anderen Stapelplätze der Hanse in London, Bergen und Nowgorod denken ${ }^{170}$ ) belegen, $\mathrm{da}$ die Errichtung eines Hospitals nicht zu den Prioritäten der sich dort etablierenden landsmannschaftlichen Bruderschaften und $/ K o l o n i e n `$ gehörte ${ }^{171}$. Während die Italiener (wobei man natürlich zwischen Genuesen, Venezianern, Florentinern und Lucchesen zu unterscheiden hat!) im Norden - wohl auch wegen der höheren Sprachbarriere - nicht selten zur sozialen Abkapselung gegenüber der sie umgebenden städtischen Gesellschaft neigten ${ }^{172}$, waren die (Nieder-)Deutschen in Flandern so gut integriert, daß ihnen der Gedanke, Hospitäler für eigene Landsleute zu stiften, wohl erst gar nicht gekommen sein mag, zumal das Gesundheitswesen in Städten wie Antwerpen schon fest unter kommunaler Aufsicht stand. Hier liegt nun das Besondere des Standortes Rom, der sich dadurch auszeichnete, daß er nicht nur ein ökonomisch interessantes Ziel von Händlern aus ganz Europa war (wie dies z.B. in Brügge oder Lyon der Fall war) und auch nicht nur - wie Santiago di Compostela - ausschließlich ein Pilgerzentrum. An den Tiber kam man meist mit mehreren Absichten! Hier lockten nicht nur die heiligen Stätten, sondern man konnte in Rom auch - denken wir nur an die vielen Möglichkeiten, die die Kurie bot -

in: Dentro la città (wie Anm. 150), S. 257-278. Vgl. zu Trient Serena LUZZI, Stranieri in città. Presenza tedesca e società urbana a Trento (secoli XV-XVIII), Bologna 2003 (Annali dell'Istituto storico italo-germanico in Trento. Monografie, 38), bes. S. 198, 211. Zu den Unterschieden von Stadt zu Stadt, die an dieser Stelle nicht vertieft werden können, vgl. jetzt ISRAEL, Fremde aus dem Norden (wie Anm. 164).

${ }^{169}$ Renée RöSSNER, Hansische Memoria in Flandern. Alltagsleben und Totengedenken der Osterlinge in Brügge und Antwerpen (13. bis 16. Jahrhundert), Frankfurt a. M. u.a. 2001 (Kieler Werkstücke, Reihe D: Beiträge zur europäischen Geschichte des späten Mittelalters, 15: Hansekaufleute in Brügge. Teil 5); EsCH, Viele Loyalitäten (wie Anm. 165).

${ }^{170} \mathrm{Vgl}$. RösSNER, Hansische Memoria (wie Anm. 169), S. 226-232.

171 Vgl. Herman LeLoux, Kirche und Caritas im Leben der Genossenschaft des Deutschen Kaufmanns zu Brügge, in: Hansische Geschichtsblätter 91 (1973) S. 34-45 (S. 44 erwähnt nur eine Hospitalsgründung in Hoek bei Rotterdam). Es wäre lohnend, die Bestandsdauer der in der Regel sehr kleinen Hospitäler der landsmannschaftlichen (Handwerks) Bruderschaften im einzelnen zu überprüfen und $\mathrm{zu}$ vergleichen. So ging die Bruderschaft der deutschen Schuster in Florenz, die Mitte des 15. Jahrhunderts ein Hospital besaß, 1502 mit zuletzt nur noch drei deutschen Mitgliedern in der Bruderschaft ihrer italienischen Kollegen auf: FRAN. CESCHI, I tedeschi e l'Arte della lana 1993, S. 260.

${ }^{172}$ ESCH, Viele Loyalitäten 1992, S. 590ff.; Giovanna PETTI BALBI, Mercanti e nationes nelle Fiandre: i genovesi in età bassomedievale, Pisa 1996 (Piccola biblioteca GISEM 7); RÖSSNER, Hansische Memoria (wie Anm. 169), S. 249-253 (auch mit Nachweisen für Integrationsbemühungen). Vgl. zur Präsenz anderer Volksgruppen (Spanier, Portugiesen, Engländer etc.) in Brügge und Antwerpen ibid. S. 243-248. Umgekehrt spielten die Fremden als Träger von Hospitälern in Genua offenbar keine Rolle: vgl. Carlo MARCHESANl, Giorgio SPERATI, Ospedali genovesi nel medioevo, Atti della Società Ligure di Storia Patria, n.s. 21, fasc. 1 (1981) (gesamter Bd.). 
〉Geschäfte und Karriere machen ${ }^{173}$. Jüngst hat man darauf hingewiesen, daß viele, die als Pilger nach Rom gekommen waren, aus religiösen Motiven in der Stadt blieben ${ }^{174}$; aber oft dürften auch ganz humane Gründe eine Rolle gespielt haben (man kann dabei mit den Vorteilen des Klimas beginnen). Andere fanden eine wirtschaftliche Nische und praktizierten ihr angestammtes Handwerk. Der karitative Auftrag war in Rom aber auch deswegen so ausgeprägt, da hier nicht Kaufleute und Transitreisende, sondern Kleriker ${ }^{175}$ die einflußreichsten Förderer der snationalen` Gemeinden waren und der Ansturm von Pilgern zumal zu den Heiligen Jahren so groß war, daß eine Lösung unabdingbar war. Ganz unberücksichtigt muß vorerst die Frage bleiben, wie viele Pilger und Reisende tatsächlich bei ihren Landsleuten oder bei den anderen größeren, besser ausgestatteten Krankenhäusern der Stadt unterkamen ${ }^{176}$. Ein Monopol bestand nicht und war auch niemals intendiert, da es die Strukturen der kleinen Hospize für die Auswärtigen überfordert hätte. Wenn selbst in Florenz (einer wichtigen Reiseetappe auf dem Weg an den Tiber) während des Jubeljahres 1450 die Präsenz von Fremden in den Krankenhäusern anstieg ${ }^{177}$, kann man ermessen, welchen Ansturm die Stadt Rom zu verkraften hatte. Und es ist be-

${ }^{173}$ Für die Vielfalt der Motive, die die Menschen nach Rom führte und die hier nur angedeutet werden kann, vgl. Michael MaTHEuS, Fremde im Rom des Mittelalters und der Renaissance, in: Fremdsein - Historische Erfahrungen, Essen 1995(Essener Unikate 6/7), S. 43-52 (mit weiterer Literatur) und Arnold EsCH, Deutsche im Rom der Renaissance: Indizien für Verweildauer, Fluktuation, Kontakte zur alten Heimat, in: Brigitte FLUG, Michael MATHEUS, Andreas REHBERG ( $\mathrm{Hg}$.), Kurie und Region. Festschrift für Brigide Schwarz zum 65. Geburtstag, Stuttgart 2005 (Geschichtliche Landeskunde, 59), S. 273-287 (jeweils mit weiterer Literatur).

174 Vgl. Anm. 116.

${ }^{175} \mathrm{Vgl}$. zum hohen Anteil der Kurialen und Kleriker an den einflußreichen Fremden in Rom Pierre HURTUBISE, La présence des »étrangers« à la cour de Rome dans la première moitié du XVI siècle, in: Forestieri e stranieri nelle città basso-medievali, Atti del seminario internazionale di studio, Bagno a Ripoli (Firenze, 4-8 giugno 1984), Florenz 1988 (Quaderni di storia urbana e rurale, 9), S. 57-80. Auf die durch die Kurie bedingte besondere Rolle Roms für die in Italien operierenden deutschen Handwerker hat schon Knut SCHULZ, Deutsche Handwerkergruppen (wie Anm. 147), S. 3-22, bes. S. 19 (mit weiterer Literatur zu anderen Städten) hingewiesen.

${ }^{176}$ Für das Florentiner Hospital S. Matteo hat Lucia SANDRI, Stranieri e forestieri nella Fi. renze del Quattrocento attraverso i libri di ricordi e di entrata e uscita degli ospedali cittadini, in: Forestieri e stranieri (wie Anm. 175), S. 149-161 festgestellt, daß von den 1276 in den Jahren von 1413 bis 1456 im Hospital Verstorbenen $18 \%$ von jenseits der Alpen stammten (davon waren $40 \%$ Deutsche, $16 \%$ Franzosen, 5,3\% Flamen etc.). Zum Pilgerwesen im Mittelalter gibt es eine kaum noch zu überblickende Literatur: vgl. hier nur Klaus HERBERS (Hg.), Stadt und Pilger. Soziale Gemeinschaften und Heiligenkult, Tübingen 1999 (JakobusStudien 10); Michael MATHEUS ( $\mathrm{Hg}$.), Pilger und Wallfahrtsstätten in Mittelalter und Neuzeit, Stuttgart 1999 (Mainzer Vorträge, 4) und Luisa D'ARIENzo, (Hg.), Gli Anni Santi nella Storia. Atti del convegno internazionale di studi (Cagliari 16-19 ottobre 1999), Cagliari 2000.

${ }^{17}$ SANDRI, Stranieri e forestieri (wie Anm. 176), S. 152. 
zeichnend für den konservativen Zug im Gesundheitswesen im päpstlichen Rom selbst des 16. Jahrhunderts, daß man noch im Vorfeld des Heiligen Jahres 1550 die Gründung neuer Hospize für Pilger der Initiative einzelner Gruppen frommer Menschen überließ und noch nicht als allgemeine `öffentliche` Aufgabe begriff ${ }^{18}$.

${ }^{178}$ SIMONCELLI, Note sul sistema (wie Anm. 4), S. 143. 